\title{
Transposable elements mediate genetic effects altering the expression of nearby genes in colorectal cancer
}

Nikolaos Lykoskoufis ( $\square$ nikolaos.lykoskoufis@unige.ch )

University of Geneva

Evarist Planet

School of Life Sciences, Ecole Polytechnique Fédérale de Lausanne (EPFL)

Halit Ongen

University of Geneva https://orcid.org/0000-0002-4197-5790

Didier Trono

School of Life Sciences, Ecole Polytechnique Fédérale de Lausanne (EPFL) https://orcid.org/00000002-3383-0401

Emmanouil T Dermitzakis

University of Geneva Medical School, Geneva, Switzerland https://orcid.org/0000-0002-9302-6490

Article

Keywords:

Posted Date: December 17th, 2021

DOI: https://doi.org/10.21203/rs.3.rs-1138340/v1

License: (c) (1) This work is licensed under a Creative Commons Attribution 4.0 International License. Read Full License 
1 Transposable elements mediate genetic effects altering the expression of nearby genes in colorectal cancer

5 Nikolaos M. R. Lykoskoufis ${ }^{1,2,3^{\star}}$, Evarist Planet ${ }^{4}$, Halit Ongen ${ }^{1,2,3}$, Didier Trono ${ }^{4}$, Emmanouil T.

6 Dermitzakis ${ }^{1,2,3^{*}}$

7

8

$9{ }^{1}$ Department of Genetic Medicine and Development, University of Geneva Medical School, 101211 Geneva, Switzerland

112 Institute for Genetics and Genomics in Geneva (iGE3), University of Geneva, 1211 Geneva, 12 Switzerland

$13{ }^{3}$ Swiss Institute of Bioinformatics, 1211 Geneva, Switzerland

$14{ }^{4}$ School of Life Sciences, Ecole Polytechnique Fédérale de Lausanne (EPFL), 1015, 15 Lausanne, Switzerland

16

17 * Corresponding authors: N.M.R Lykoskoufis: nikolaos.lykoskoufis@unige.ch and E.T.D:

18 emmanouil.dermitzakis@unige.ch

19 Department of Genetic Medicine and Development, University of Geneva,

201 rue Michel-Servet, 1211 Geneva, Switzerland

21 


\section{ABSTRACT}

25 Transposable elements (TEs) are interspersed repeats that contribute to more than half of the human genome, and TE-embedded regulatory sequences are increasingly recognized as major components of the human regulome. Perturbations of this system can contribute to tumorigenesis, but the impact of TEs on gene expression in cancer cells remains to be fully assessed. Here, we analyzed 275 normal colon and 276 colorectal cancer (CRC) samples

30 from the SYSCOL colorectal cancer cohort and discovered 10,111 and 5,152 TE expression

31 quantitative trait loci (eQTLs) in normal and tumor tissues, respectively. Amongst the latter, 376 were exclusive to $\mathrm{CRC}$, likely driven by changes in methylation patterns. We identified that transcription factors are more enriched in tumor-specific TE-eQTLs than shared TEeQTLs, indicating that TEs are more specifically regulated in tumor than normal. Using Bayesian Networks to assess the causal relationship between eQTL variants, TEs and genes, we identified that 1,758 TEs are mediators of genetic effect, altering the expression of 1,626 nearby genes significantly more in tumor compared to normal, of which 51 are cancer driver genes. We show that tumor-specific TE-eQTLs trigger the driver capability of TEs subsequently impacting expression of nearby genes. Collectively, our results highlight a global profile of a new class of cancer drivers, thereby enhancing our understanding of tumorigenesis and providing potential new candidate mechanisms for therapeutic target development. 


\section{INTRODUCTION}

45 Understanding the mechanisms underlying tumorigenesis has been one of the main research questions in cancer biology. While somatic mutations, chromosomal rearrangements and gene amplification are the three main hallmarks driving cancer progression, they are unable to provide a complete explanation of tumorigenesis. Recent discoveries have demonstrated that transposable elements (TEs) have contributed to the evolution of gene regulation and can alter the landscape of gene expression in development and disease [1-5]. Transposable elements (TEs) are interspersed repeats that contribute more than half of the human genome. TEs, more specifically TE-embedded regulatory sequences (TEeRS) are broadly active during the phases of genome reprogramming that occur in the germline and the early embryo, and then controlled by epigenetic mechanisms that still allow their finely orchestrated participation in biological events as diverse as brain development, immune responses, and metabolic control. The aberrant re-activation of TEeRSs is observed under certain conditions and disease states, notably cancer [6-8]. Transcription is defined by the coordinated activity of regulatory elements which are modulated by genetic variation. Thus, we speculate that transposable element expression is influenced by regulatory non-coding variants, also called expression Quantitative Trait Loci (eQTLs), known to contribute to the onset and progression of complex diseases like cancer $[9,10]$. To build on this concept, we set out to analyze the interplay between regulatory variants (eQTLs), transposable elements and gene expression to characterize the genetic perturbation of TE and gene expression in cancer. To this end, we integrated genome-wide genotyping data (genotype array) and transcriptomic profiles (bulk RNA-sequencing) from the Systems Biology of Colorectal Cancer (SYSCOL) cohort comprising of 275 and 276 normal and tumor samples, respectively.

67 


\section{Quantifying transposable elements (TEs) and gene expression}

To measure the expression of TEs in CRC, we examined transcriptomes obtained by RNAseq from 275 normal and 276 CRC samples from the SYSCOL cohort [11]. We quantified TE and gene expression using an in-house curated TE annotation list originating from the RepBase database [12] that contains approximately 4.6 million individual TE loci. These annotations were merged with gene annotation from ensembl (v19). Filtering for uniquely mapped reads (Methods) to obtain robust estimates of TE expression resulted in 51,320 TEs and 17,360 genes (protein coding and lincRNAs). We observed that the majority of expressed TEs present in our dataset are SINEs (Alu and MIR), LINEs (L1 and L2) as well as different subfamilies of Long Terminal Repeats (LTRs) and DNA transposons. However, when we looked at the proportion of expressed TEs per subfamily, SVA and ERVK were most prominent (Figure 1A, Supplementary figure 1). Additionally, we used available data from Encode [13] and miRbase [14] to generate a list of regulatory regions and discovered that 13,656 expressed TEs overlapped with at least one previously identified regulatory element. We also discovered that expressed TEs are significantly enriched for most regulatory regions, except for enhancers, compared to non-expressed TEs (Supplementary table 1; Figure 1B, Supplementary figure 2) highlighting their potential role in gene expression regulation.

\section{Transposable elements are under strong genetic control}

Using TE expression quantifications and genotype data we first sought to assess the impact

91 of inter-individual genetic variation on TE expression. We conducted cis-eQTL analysis

92 followed by a forward backward stepwise conditional analysis (Methods) and discovered a

93 total of 10'111 and 5'152 TE-eQTLs as well as 6'856 and 1'539 gene eQTLs in normal and

94 tumoral tissue, respectively (Supplementary Figure 3,4; Supplementary Tables 2,3). 
95 Similarly to gene-eQTLs, TE-eQTLs displayed stronger effects and density closer to the 96 transcription start site (TSS) in both normal and tumor samples (spearman rho=-0.34, $\mathrm{P}<2.2 \mathrm{e}-$

9716 in normal, spearman rho=-0.27, $\mathrm{P}<2.2 \mathrm{e}-16$ in tumor) (Figure 2A-B), yet were more proximal to the TSS compared to gene-eQTLs (Wilcoxon $\mathrm{P}=7.6 \mathrm{e}-11$ in normal; Wilcoxon $\mathrm{P}=3.3 \mathrm{e}-05$ in tumor; Supplementary Figure 5). We observed that TEs displayed fewer independent eQTLs per TE than genes (Figure 2C-D) while the minor allele frequencies of

101 TE- and gene-eQTL variants were similar (Supplementary Figure 6). Proximal distance of 102 TE-eQTLs to TSS and the smaller number of independent signals per TE could be due to 103 smaller evolutionary time of TE regulatory landscapes in the human genome compared to 104 genes, making proximal effects much more likely.

105 Given previously established roles of tumor-specific gene-eQTLs in tumorigenesis [11], we aimed next at investigating whether tumor-specific TE-eQTLs could similarly contribute as cancer driving factors. To this end, we used linear mix models with an interaction term between

108 variant and tissue (normal/tumor). We discovered that $376(7.3 \%)$ of the tumor TE-eQTLs are 109 tumor-specific and 1,685 (17\%) of the normal TE-eQTLs are normal-specific, with 524 TE110 eQTLs shared between both settings (Figure 3A; Supplementary Table 5). For genes, we 111 found 101 (6.5\%) tumor gene-eQTLs to be tumor-specific and 897 (13\%) normal gene-eQTLs 112 to be normal-specific, of which 169 were shared (Supplementary Figure 7A; Supplementary

113 Table 4). Shared TE- and gene-eQTLs were closer to the TSS of TEs/genes compared to 114 tissue-specific eQTLs (Wilcoxon $\mathrm{P}<2.2 \mathrm{e}-16$ ) (Figure 3B, Supplementary figure 7B).

115 Additionally, we observed that shared eQTLs conserved their effect in both normal and tumor

116 (Figure 3C, Supplementary figure 7C). These results indicate that TE expression is under 117 strong genetic control and that non-coding germline variants act as drivers of TE expression 118 in cancer as similarly observed for gene expression [11]. 
121 Transcription factors regulate TE expression more specifically in

122 tumor

123 To corroborate the biological relevance of the discovered TE-eQTL variants we performed

124 functional enrichment analysis of TE and gene eQTLs in normal and tumor using available

125 ChIP-seq data from the Ensembl Regulatory Build [15] (methods). We found significant

126 enrichment for many TF binding sites overlapping the eQTL loci highlighting the functional

127 relevance of the variants discovered (Figure 4A-B; Supplementary Figure 8,9;

128 Supplementary Tables 6,7). We discovered 5 TFs and 15 TFs that displayed stronger

129 enrichment for TE eQTLs compared to gene eQTLs in normal and tumor, respectively. The

130 TF most enriched over TE-eQTLs in normal tissues was ZNF274, a Krüppel-associated box

131 (KRAB) domain-containing zinc-finger protein (KZFP), whereas the most enriched over tumor

132 TE-eQTLs was TRIM28, the master corepressor that is recruited by the KRAB domain of many

133 TE-binding KZFPs and serves as a scaffold for a heterochromatin-inducing complex capable

134 of repressing TEs via histone H3 Lys9 trimethylation (H3K9me3), histone deacetylation and

135 DNA methylation [16, 17]. Additionally, BDP1 and BRF1, two subunits of the RNA polymerase

136 III transcription initiation factor, were more enriched over TE-eQTLs compared to gene eQTLs

137 highlighting potential transcription of Alu or MIR TEs of the SINE family [18]. These results

138 corroborate the biological relevance of TE eQTLs and point to possible transcription and

139 repression of certain TEs.

140 To assess the differential effects of tumor-specific versus shared eQTLs, we performed

141 functional enrichment analyses using available ChIP-seq data from LoVo colorectal cancer

142 cells [19] (methods). We observed that in the case of genes, all tested TFs had a stronger

143 enrichment for shared compared to tumor-specific eQTLs, indicating that these TFs are

144 regulating gene expression in both the normal and tumor state. (Supplementary Figure 10,

145 Supplementary Table 8). In contrast, we found 80 TFs displaying stronger enrichment for

146 tumor-specific versus shared TE-eQTLs, pointing to tumor-specific TE regulation (Figure 4C;

147 Supplementary Figure 11; Supplementary Table 9). Of these, 39 were upregulated and 34 
148 downregulated in tumors (7 were missing from our expression data and could not be tested

149 for differential expression analysis), but we did not observe any significant correlation between

150 the tumor-specific TE-eQTL enrichment to shared TE-eQTL enrichment ratio and fold change

151 in the expression of the corresponding transcription factors (Pearson $R=-0.18, p$-value $=$

152 0.083; Supplementary Figure 12). Thus, differential expression of these TFs is not driving 153 the tumor-specific TE-eQTL effects. However, 59 of the 86 tumor-specific TE-eQTLs 154 overlapping the binding sites of the 80 aforementioned TFs are not significantly associated 155 (FDR $=5 \%)$ with any nearby $( \pm 1 \mathrm{Mb}$ from TSS) TE or gene in normal, indicating that these 156 regulatory regions are inactive in the normal state (Supplementary Figure 13). Additionally, 157 we compared methylation levels between normal and tumor samples for the tumor-specific 158 and shared eQTLs and observed significantly increased (Wilcoxon rank sum test $p$-value $=$ 1590.017 for TEs and $p$-value $=0.00097$ for genes) methylation over tumor-specific compared to 160 shared eQTLs for both gene and TEs (Figure 3D; Supplementary figure 7D).

161 Altogether these results suggest that many TFs are regulating TE expression. The inactivity 162 of some of the TE eQTLs in normal and the significant changes in methylation between tumor163 specific and shared TE-eQTLs indicate that regulatory switches involving the recruitment of 164 these TFs might underlie the effects of tumor-specific TE eQTLs.

\section{Transposable elements as mediators of genetic effects onto genes}

167 Having established that TEs are under genetic control, we next sought to assess the causal 168 relationship between eQTL variants, TEs and genes and discover the extent to which TEs act 169 as drivers of gene expression in tumor. To this end, we focused on regulatory variants affecting 170 both TEs and genes and detected these in an unbiased manner by first associating TEs with 171 genes using a similar approach to QTL mapping. Next, we quantified the identified 21,263 TE172 gene pairs found in normal samples and 144,289 TE-gene pairs found in tumor at 1\% FDR 173 and used this newly quantified TE-gene pairs to find all eQTL-TE-gene triplets by performing 174 a standard eQTL analysis (Methods; Supplementary figure 14-16). At 5\% FDR, we 
175 discovered 12,379 and 9,714 triplets in normal and tumor, respectively, for which we inferred

176 the most likely causal relationship using Bayesian networks (Methods) [20-22]. We tested

177 three models, (i) the causal model where the eQTL variant affects TE expression and then

178 gene expression, (ii) the reactive model where the eQTL variant affects gene expression and

179 then TE expression and (iii) the independent model where the eQTL variant affects

180 independently TE and gene expression (Supplementary figure 17). We observed

181 significantly more causal models in tumor (47\%) compared to normal (22\%) (Fisher p-value

$182<2 \mathrm{e}^{-16}$ ) indicating that TEs are causal for gene expression predominantly in tumor and to a

183 lesser extent in normal (Figure 5A, B; Supplementary figure 18; Supplementary Table

184 10,11). We also show that the proportion of causal models correlated with the genomic 185 position of the TE with respect to the gene; intronic TEs tended to be reacting to gene 186 expression whereas TEs outside the gene body tended to be causal. Interestingly, there were significantly more causal scenarios when the eQTL variant lied within the TE, rather than

188 outside (Fisher $p$-value $<2 \mathrm{e}^{-16}$ ) pinpointing to direct regulatory effects of the TE onto gene 189 expression (Supplementary figure 19). Altogether, these results show that TEs are 190 significantly more causal for changes in gene expression in tumor than in normal tissue.

192 Transposable elements are drivers of gene expression during 193 tumorigenesis

194 These results suggested that genetic variations in TE expression might drive tumorigenesis.

195 To test this hypothesis, we considered the union of all triplets, i.e. the eQTL variant, TE and 196 gene expression, discovered across tumor and normal tissue and using the same BN 197 approach as previously mentioned, we inferred the causal relationship between the triplets in 198 both states (methods). We similarly looked for shared triplets across the 12,379 normal and 1999,714 tumor triplets (eQTL-TE-gene triplets are the same in both states or the eQTL for TEgene pair is in high $\left.\operatorname{LD}\left(R^{2}>=0.9\right)\right)$. In both shared and union triplets, we observed a significant increase in the causal model in tumor (Fisher Exact Test $\mathrm{p}$-value $<2.2 \mathrm{e}^{-16}$ for shared and union 
202 triplets) mainly due to independent models and to a lesser extent reactive model shifting to

203 causal. (Supplementary figure 20; Supplementary Table 12,13). Focusing on the 9,714

204 tumor triplets, we discovered 2,651 (28\%) triplets that switched to a causal model in tumor 205 compared to normal, highlighting regulatory changes whereby TEs impacted the expression 206 of nearby genes (Figure 5C). These 2,651 triplets constituted of 1,758 unique TEs impacting 2071,626 unique genes. Interestingly, we observed that TEs switching to causal were significantly 208 up-regulated compared to TEs that did not switch models between normal and tumor or that 209 switched but not to causal (Wilcoxon p-value 2.9e-6; Supplementary Figure 21). These 210 results suggest that upregulation of TEs could give rise to their gene expression driver 211 capability.

212 While expression of most TEs was positively correlated with the expression of the associated 213 gene in tumor $(n=2,639)$ (Figure 5D), only a few showed negative correlation $(n=12)$. Of the significant tumor TE-gene pairs tested in normal colon, we observed that 940 maintained the

215 same effect (in terms of size and direction) whereas 34 showed an opposite effect in tumor 216 samples. Interestingly, of the 1,626 genes, 51 were cancer driver genes (CDG) (5 CRC 217 specific; based on Cancer Gene Census [23]) but we did not find a significant enrichment of

218 CDGs in triplets switching to causal compared to all other tumor triplets (Fisher exact test p219 value $=\mathbf{0 . 2 9 0 3}$; odds-ratio $=\mathbf{1 . 2 7 4}$ ). For 34 out of the $51 \mathrm{CDGs}$, we did not find a significant 220 correlation between their expression and the expression of the corresponding TEs in normal 221 samples pinpointing that these TEs have no impact on these genes in the normal state. Taken 222 together, these results suggest an important role of TEs as drivers of gene expression during 223 tumorigenesis. 
226 Non-coding germline variants activate driver TEs during

\section{7 tumorigenesis}

228 We investigated whether any of the 9,714 tumor triplets were constituted of any previously

229 identified tumor-specific or shared TE-eQTLs and assess how the model likelihood changed

230 between normal and tumor. We identified 363 and 128 tumor triplets constituted of a shared

231 or a tumor-specific TE-eQTL, respectively (Figure 6A-B) and observed that the 128 tumor

232 triplets constituted with a tumor-specific TE-eQTL are significantly enriched for triplets

233 switching to causal compared to the 363 tumor triplets constituted with a shared TE-eQTL

234 (Fisher Exact test p-value = 3.147e-05; Odds-ratio=2.4) (Figure 6B). Additionally, we 235 observed that for 115 triplets with tumor-specific TE-eQTLs, the eQTL variant was not a 236 significant eQTL for the corresponding gene in the triplet (Figure 6C), highlighting that the 237 eQTLs get activated in the tumor state influencing TE expression that subsequently impact 238 gene expression. Altogether, these results suggest that tumor-specific TE-eQTLs contribute

239 to tumorigenesis by impacting genes through TEs, adding additional proof that germline 240 variants can be contributing to tumorigenesis.

\section{Driver TEs act as alternative promoters for genes in cancer}

243 It has been shown that TEs could impact gene expression by acting as alternative promoters

244 for nearby genes and creating chimeric transcripts (transpochimeric transcripts (tcGTs)) [24, 25]. To assess whether any of the tumor triplets with causal TEs were affected by tcGT events,

246 we looked for cases where transcripts started from a TE and spliced into a single or multiple

247 nearby genes (methods). We only kept tcGTs made up of the same TE and gene as in the

2489,714 tumor triplets and that were significantly more abundant in tumor samples compared to

249 normal samples using a Fisher exact test. At 5\% FDR, we discovered 117 tcGTs present in 250138 tumor triplets. Of these, 72 were causal and 46 switched to causal from normal to tumor. 251 Interestingly, we detected tcGT events with a known tumor suppressor gene RNF43 and two 
252 oncogenes ETS2 and SLCO1B3 supporting the extensive contribution of TEs during 253 tumorigenesis.

\section{DISCUSSION}

255

256

257

258

259

Transposable elements are important contributors to tumorigenesis and provide supplementary means by which gene expression can be altered in cancer. While many studies have used a hypothesis-driven approach and focused at specific TEs or their subfamilies for discovering TEs that alter the expression of nearby genes in cancer [26-28], applying a genome-wide scan could allow to obtain a better picture of the effects of TEs on gene expression during tumorigenesis.

Here, we present a global profile of tumor drivers and show that TEs are highly prevalent mediators of genetic effects on genes altering their expression, specifically in tumor. By combining genome and transcriptome data together we, show that TEs are under tight genetic control and discover that transcription factors regulate TE expression much more in tumor than in normal. By looking at the interplay between eQTL variants, transposable elements, and gene expression, we are able to dissect eQTL effects and show that for several genes, the genetic effect of an eQTL is passed on genes through TEs which act as mediators and drive gene expression. We observe this to occur significantly more in cancer than in normal and show that the majority of TEs increase the expression of affected nearby genes. Interestingly, we discover that TEs affecting known cancer driver genes in cancer have for most part no significant effect on these genes in normal suggesting a tumor-specific effect of these TEs. Additionally, in our study we show that alongside predisposing alleles and somatic mutations, germline variants are crucial contributors to tumorigenesis as these allow for transcriptional changes to occur at the level of TEs that in turn result in altered expression of nearby genes in cancer as shown previously [11].

While we focused on TEs impacting the expression of nearby genes in an independent manner, it is highly plausible that synergistic effects occur from both cis- and trans- acting 
279 TEs. Performing such an analysis could give a fuller picture of the regulatory network behind

280 the regulation of gene expression through TE effects, requiring, however, a high sample size

281 for sufficient statistical power. Nevertheless, because of the highly repetitive nature of

282 transposable element sequences and their evolutionary relatedness among TE families,

283 mapping short reads originating from TEs is a real challenge [18, 29]. Our RNA-seq dataset

284 having a read length of $49 \mathrm{bp}$, it is highly possible that we did not map all expressed TEs

285 subsequently leading to missing information, as shown previously [18, 29]. Future studies

286 where RNA-sequencing is performed with longer read lengths could allow for better mapping

287 of expressed TEs and give us a fuller picture of the number of these driver TEs in cancer.

288 Altogether, we have outlined that TEs are important mediators of genetic effects onto genes

289 that could potentially be used as risk factors or new therapeutic targets for future drug 290 development and aid in cancer treatment. 


\section{METHODS}

293

294

295

296

297

298

299

300

301

302

303

\subsection{SYSCOL dataset}

The Systems Biology of Colorectal cancer (SYSCOL) dataset contains data from genotypes and RNA-sequencing for matched normal-tumor samples (i.e., both tumor and normal samples originate from the same patient). Samples that had genotype data and molecular phenotype quantifications from tumor and normal (normal adjacent to tumor) tissue were analyzed, yielding 275 normal samples and 276 tumor samples. In case of multiple tumor samples from the same patient, only samples with quantifications from the most advanced tumor were kept.

\subsection{Genotypes}

We used imputed genotypes and only kept variants with a minor allele frequency $(M A F)>=5 \%$, yielding a total of $6,132,240$ variants that were used for all downstream analyses.

\subsection{Transcriptome quantifications}

\subsubsection{Read mapping}

SYSCOL samples were sequenced using 49bp, 75bp and 100bp read lengths. We first started by trimming all $75 \mathrm{bp}$ and $100 \mathrm{bp}$ reads down to $49 \mathrm{bp}$ to reduce any bias in downstream analysis stemming from read length. All trimmed samples were mapped to the human reference genome (hg37) using hisat2 [30]

\subsubsection{Transposable elements (TE) and genes quantifications}

Gene and transposable element counts were generated using the featureCounts software [31]. We provided a single annotation file in gtf format to featureCounts containing both genes and transposable elements. This prevents any read assignation ambiguity to occur. For transposable elements, we used an in-house curated version of the Repbase database [12] where we merged fragmented LTR and internal segments belonging to a single integrant. We only used uniquely mapped reads for gene and TE counts. Molecular phenotypes that did not have at least one sample with 20 reads and for which the sum of reads across all samples 
318 was lower than the number of samples, were discarded. Furthermore, we normalized

319 molecular phenotypes (TEs and genes) for sequencing depth using the TMM methodology as

320 implemented in the limma package of Bioconductor [32] and used gene counts as library size

321 for both TEs and genes. Finally, we removed any molecular phenotype that had more than

$32250 \%$ of missing data (zeros) in tumor and normal samples separately and took the union of

323 molecular phenotypes, yielding $17^{\prime} 360$ genes and $45^{\prime} 717$ TEs for a total of $63^{\prime} 077$ molecular

324 phenotypes.

\subsubsection{Normalization of molecular phenotypes}

The observed variability in molecular phenotypes from RNA-sequencing data can be of biological or technical origin. To correct for technical variability, while retaining biological variability, we residualised the molecular phenotype data for the covariates as described below:

1. To correct for population stratification that is observed between the SYSCOL samples, we used Principal Component analysis (PCA) results obtained from genotypes of SYSCOL patients. We only retained the first three principal components (PCs) as covariates.

2. In order to capture experimental/technical variability in the expression data, we performed PCA, centering and scaling, using pca mode from QTLtools software package [33]. To ascertain the number of PCs that capture technical variability, we used QTL mapping (see method 3.4.1 for the description of QTL mapping) to identify the best eQTL discovery power in both tumor and normal samples. To this end, we carried out multiple rounds of eQTL mapping for tumor and normal samples separately, each time using the 3 PCs from genotypes and incrementally adding $0,1,2,5,10,20$, $30,40,50,60$ and 70 PCs as covariates. This approach resulted in identifying 30 PCs in tumor and normal samples for maximizing eQTL discovery.

343 In total, 33 covariates were regressed out from tumor and normal sample expression data 344 using QTLtools correct mode [33]. We additionally rank-normalized on a per phenotype basis 
across all samples such that quantifications followed normal distribution with mean 0 and

346 standard deviation $1 \mathrm{~N}(0,1)$ using QTLtools --normal option [33].

We used microarray based DNA methylation data from the SYSCOL project and a similar approach to a previous study to find differential methylation of eQTLs [11]. In brief, we calculated the absolute value difference of the medians of normalized methylation probe betas in normal and tumor that we call median differential methylation. We then compared the distribution of there medians in tumor-specific TE and genes eQTLs vs. the shared TE and gene eQTLs and calculated a P-value using the Mann Whitney U test. P-values were corrected for multiple testing using the $R /$ qvalue package with a given FDR threshold of $5 \%$.

\subsection{Differential TE/gene expression analysis}

The DESeq2 R package [34] was used in calculating differentially expressed genes and TEs.

We normalized the raw TE/gene counts within the DESeq2 package using the sequencing date, GC mean and insert size as covariates. The differential expression P-values were corrected for multiple testing using an FDR threshold of $5 \%$.

\subsection{Transcriptome QTL analysis}

All analyses were performed separately for normal and tumor samples. We used imputed genotypes with MAF >=5 \%, gene expression data with normalized counts per million (CPMs) (as described above) for both eQTL and conditional eQTL mapping.

\subsubsection{Expression Quantitative Trait Loci (eQTL) mapping}

For eQTL mapping, we used cis mode of the QTLtools software package [33]. For each molecular phenotype:

1. We counted all genetic variants in a $1 \mathrm{Mb}$ window $(+/-1 \mathrm{Mb})$ around the transcription start site (TSS) of the phenotype and tested all variants within this 
window for association with the phenotype. We only retained the best hits which are defined as the ones with the smallest nominal $p$-value.

2. Next, we used permutations to adjust the nominal p-values for the number of variants tested. More specifically, we randomly shuffled the quantifications of the phenotypes 1'000 times and retained only the most significant associations. This created a null distribution of 1'000 null p-values. Then, we fitted a beta distribution on the null distribution and used the resulting beta distribution to adjust the nominal p-value. Principally, this strategy allows to quantify the chance of getting a smaller p-value than the nominal one in random datasets.

This effectively gave us the best variant in cis together with the corresponding adjusted pvalue of association for each molecular phenotype. Finally, to correct for the number of phenotypes being tested we used False Discovery Rate (FDR) correction approach. More specifically, we used the R/qvalue package [35] to perform genome-wide FDR correction which ultimately facilitated to extract all phenotype-variant pairs that are significant at a predetermined FDR threshold, 5\% FDR in this case.

\subsubsection{Conditional analysis for $e Q T L$ mapping}

The cis mode informs us on the best phenotype-variant pair only. Given that the expression of molecular phenotypes can be affected by multiple cis eQTLs, we proceeded with conditional analysis to discover all eQTLs with independent functional effects on a given phenotype. Principally, new discoveries are made after conditioning on previous ones. Again, cis mode in the QTLtools software package was used [33]. In brief, after running permutations (method 1.4.1) for each phenotype, we calculated a nominal $p$-value threshold of being significant. We first determined the adjusted p-value threshold that corresponds to the targeted FDR level and then used the beta quantile function to go from adjusted $p$-value to a specific nominal $p$-value 
threshold. For conditional analysis, forward-backward methodology is used to discover all

400 independent QTLs and to identify the most likely candidate variants, while at the same time controlling for a given FDR (5\% FDR in this case). We only kept the top variant for each signal.

\subsubsection{Tissue-specific and shared eQTL analysis}

404

To discover tissue specific and shared eQTLs, we used the eQTL results obtained after running the conditional pass. In total, we tested 17 '077 eQTLs to discover normal-specific eQTLs and 6'591 to discover tumor-specific eQTLs. To do that, we used linear mix models using an interaction term between dosage and tissue (i.e tumor or normal) to test whether the slopes in normal and tumor are significantly different. Linear mix models are needed here because normal and tumor samples are originating from the same patient thus genotypes will

411 be identical. We did this for tumor and normal eQTLs separately. Then we performed multiple

412 test correction using the R/qvalue package [35] with a given FDR threshold of $5 \%$. Additionally,

413 for all significant results at 5\% FDR, if eQTL slopes (slopes given from conditional QTL

414 mapping using QTLtools) in normal and tumor had the same direction, then we only kept the ones where the SNP-phenotype association in the opposite tissue was not nominally significant $(P>0.05)$ as given by the cis nominal pass mode in the QTLtools package [33].

Shared eQTLs are defined as the ones where the P-value for the interaction term is not significant but need to be significant eQTLs (5\% FDR) in both normal and tumor as assessed by the conditional QTL mapping.

\subsubsection{Functional enrichment analysis}

422 To compare the QTL variants to a null distribution of similar variants without regulatory 423 association, we sampled for each eQTL variant 100 random regulatory genetic variants 424 matching for relative distance to TSS (withing 2.5kb) and minor allele frequency (within $2 \%$ ) 425 and only kept variants that are not eQTLs for any other TE or gene (nominal p-value > 0.05). 
426 The enrichment for a given category was calculated as the proportion between the number of

427 regulatory associations in a given category and all regulatory variants over the same 428 proportion in the null distribution of variants. The $p$-value for this enrichment is calculated with 429 the Fisher exact test. Finally, we corrected for multiple testing using an FDR threshold of 5\% 430 using the "p.adjust" function in the R programming language.

431 We used two different datasets for the functional enrichment. For gene and TE eQTLs in normal and tumor, we used available transcription factor (TF) ChIP-seq data from Ensembl

433 Regulatory Build [15]. For each TF, we combined all peaks from all available cell-types.

434 Regarding the tumor-specific vs. shared TE and gene eQTLs, we used available ChIP-seq 435 data from the colorectal cancer LoVo cell line [19].

\section{Testing for associations between TEs and genes}

437 To discover associations between TEs and genes, we proceeded in a similar way to what we 438 did for QTL mapping (method 1.4.1). Effectively, we used TE expression as our "genotypes" 439 and genes as our phenotype. Then, we corrected for multiple testing using the R/qvalue 440 package with a given FDR of $1 \%$. We then estimated the nominal $p$-value thresholds for each 441 phenotype being tested as described in (method 1.4.2) with a given FDR of $1 \%$. Given the nominal threshold we get for each gene, we then extracted all TEs with an association P-value below this threshold which could give multiple TEs for a gene in some cases.

\subsection{Quantifying TE-gene pairs}

447 To quantify each of TE-gene pairs that have been found to be significant, we used a dimensionality reduction approach based on PCA as previously described [22]. Specifically, for each TE-gene pair, we aggregated gene expression together with TE expression by using

450 the coordinates on the first PC. This effectively built a quantification matrix with rows and 451 columns corresponding to the number of TE-Gene pairs and individuals, respectively. All 
quantifications have been rank-normalized on a per phenotype basis so that the values match a normal distribution $\mathrm{N}(0,1)$. This prevents outlier effects in downstream association testing. This is all implemented in the clomics software package [22].TE

\subsection{Causal inference by Bayesian networks for QTL-TE-Gene triplets}

Bayesian networks (BNs) are a type of probabilistic graphical model that uses Bayesian inference to compute probabilities. BNs aim to model conditional dependencies and therefore causation by representing conditional dependencies as edges and random variables as nodes in a directed acyclic graph. The flow of information between two nodes is reflected by the direction of the edges, giving an idea of their causal relationship. BNs have been previously used in a genetic framework [20] to get insight into the most likely network from which the observed data originates.

In $\mathrm{BNs}$, the joint probability density can be divided into marginal probability functions and conditional probability functions for the nodes and edges, respectively. Additionally, BNs satisfy the local Markov property where each variable is conditionally independent of its nondescendants given its parent variables. In the context of this study, we used BNs to learn the causal relationships between triplets of variables, each one containing a genetic variant, a transposable element and a gene. In practice, only three distinct network topologies where relevant to the hypotheses we wanted to test (Supplementary figure 12). More specifically, we looked at:

1. The causal scenario where the genetic variant affects first the TE and then the gene.

2. The reactive scenario where the genetic variant affects the gene first and then the TE. independently.

Of note, we only retained network topologies that assume that the signal systematically originates from the genetic variant. In practice, we applied BNs on data that was obtained from 
running an QTL mapping using the TE-gene pairs using a similar approach to QTL mapping

480 described above (Method 1.4.1) and only kept significant results at 5\% FDR which 481 corresponds to 11'425 QTL-TE-gene triplets in normal and 9'488 QTL-TE-gene triplets in 482 tumor.

483 For each triplet, we build a $275 \times 3$ matrix in normal and $276 \times 3$ matrix in tumor containing normalized quantifications and used it to compute the likelihood of the $3 \mathrm{BN}$ topologies using the $R /$ bnlearn package (Version 4.5) [36]. As a last step, we went from likelihoods to posterior probabilities by assuming a uniform prior probability on the three possible topologies. Posterior probabilities where used for all BN-related analyses.

\subsection{Transpochimeric transcripts analysis}

490

491 First, a per sample transcriptome was computed from the RNA-seq bam file using StringTie 492 [37] with parameters $-\mathrm{j} 1-\mathrm{c} 1$. Each transcriptome was then crossed using BEDTools [38] to 493 both the ensembl hg19 coding exons and curated RepBase [12] to extract TcGTs for each 494 sample. Second, a custom python program was used to annotate and aggregate the sample 495 level TcGTs into counts per groups (normal, tumor). In brief, for each dataset, a GTF 496 containing all annotated TcGTs was created and TcGTs having their first exon overlapping an 497 annotated gene or TSS not overlapping a TE were discarded. From this filtered file, TcGTs 498 associated with the same gene and having a TSS $100 \mathrm{bp}$ within each other were aggregated. 499 Finally, for each aggregate, its occurrence per group was computed. 
502 AUTHOR CONTRIBUTIONS

503 N.M.R.L, H.O and E.T.D designed the study. N.M.R.L analyzed the data and wrote the 504 manuscript and N.M.R.L, H.O, D.T and E.T.D interpreted the results. E.P shared the 505 quantifications data.

506

507 COMPETING INTERESTS

508 Emmanouil T. Dermitzakis is currently an employee of GSK. The work presented in this 509 manuscript was performed before he joined GSK. All other authors declare no competing 510 interests.

511

\section{ACKNOWLEDGMENTS}

513 The computations were performed at the University of Geneva on the Baobab cluster. This

514 work was supported by grants from Louis-Jeantet Foundation support (to E.T.D) and SNSF

515 grant (to E.T.D). The funders had no role in study design, data collection and analysis, decision

516 to publish, or preparation of the manuscript.

517

518 


\section{REFERENCES}

$520 \quad$ 1. Xie, M., et al., DNA hypomethylation within specific transposable element families 836.

2. Sundaram, V., et al., Widespread contribution of transposable elements to the innovation of gene regulatory networks. Genome research, 2014. 24(12): p. 19631976.

3. Rebollo, R., M.T. Romanish, and D.L. Mager, Transposable elements: an abundant and natural source of regulatory sequences for host genes. Annual review of genetics, 2012. 46: p. 21-42.

4. $\quad$ Batut, P., et al., High-fidelity promoter profiling reveals widespread alternative promoter usage and transposon-driven developmental gene expression. Genome research, 2013. 23(1): p. 169-180.

5. Chuong, E.B., N.C. Elde, and C. Feschotte, Regulatory activities of transposable elements: from conflicts to benefits. Nature Reviews Genetics, 2017. 18(2): p. 71.

6. Hon, G.C., et al., Global DNA hypomethylation coupled to repressive chromatin domain formation and gene silencing in breast cancer. Genome research, 2012. 22(2): p. 246-258.

7. Baylin, S.B. and P.A. Jones, A decade of exploring the cancer epigenome-biological and translational implications. Nature Reviews Cancer, 2011. 11(10): p. 726-734.

8. Esteller, M., Cancer epigenomics: DNA methylomes and histone-modification maps. Nature reviews genetics, 2007. 8(4): p. 286-298.

9. Grundberg, E., et al., Mapping cis- and trans-regulatory effects across multiple tissues in twins. Nat Genet, 2012. 44(10): p. 1084-9.

10. Lappalainen, T., et al., Epistatic selection between coding and regulatory variation in human evolution and disease. Am J Hum Genet, 2011. 89(3): p. 459-63.

11. Ongen, H., et al., Putative cis-regulatory drivers in colorectal cancer. Nature, 2014. 512(7512): p. 87.

12. Bao, W., K.K. Kojima, and O. Kohany, Repbase Update, a database of repetitive elements in eukaryotic genomes. Mobile Dna, 2015. 6(1): p. 11.

13. Consortium, E.P., An integrated encyclopedia of DNA elements in the human genome. Nature, 2012. 489(7414): p. 57.

14. Kozomara, A., M. Birgaoanu, and S. Griffiths-Jones, miRBase: from microRNA sequences to function. Nucleic acids research, 2019. 47(D1): p. D155-D162.

15. Zerbino, D.R., et al., The ensembl regulatory build. Genome biology, 2015. 16(1): p. $1-8$.

16. Bourque, G., et al., Ten things you should know about transposable elements. Genome biology, 2018. 19(1): p. 1-12.

17. Imbeault, M., P.-Y. Helleboid, and D. Trono, KRAB zinc-finger proteins contribute to the evolution of gene regulatory networks. Nature, 2017. 543(7646): p. 550-554.

18. Lanciano, S. and G. Cristofari, Measuring and interpreting transposable element expression. Nature Reviews Genetics, 2020: p. 1-16.

19. Yan, J., et al., Transcription factor binding in human cells occurs in dense clusters formed around cohesin anchor sites. Cell, 2013. 154(4): p. 801-13.

20. Gutierrez-Arcelus, M., et al., Passive and active DNA methylation and the interplay with genetic variation in gene regulation. Elife, 2013. 2: p. e00523.

21. Zhu, J., et al., Integrating large-scale functional genomic data to dissect the complexity of yeast regulatory networks. Nature genetics, 2008. 40(7): p. 854-861. 
22. Delaneau, O., et al., Chromatin three-dimensional interactions mediate genetic effects on gene expression. Science, 2019. 364(6439).

23. Sondka, Z., et al., The COSMIC Cancer Gene Census: describing genetic dysfunction across all human cancers. Nature Reviews Cancer, 2018. 18(11): p. 696-705.

24. Friedli, M. and D. Trono, The developmental control of transposable elements and the evolution of higher species. Annual review of cell and developmental biology, 2015. 31: p. 429-451.

25. Jang, H.S., et al., Transposable elements drive widespread expression of oncogenes in human cancers. Nature genetics, 2019. 51(4): p. 611-617.

26. Strissel, P.L., et al., Reactivation of codogenic endogenous retroviral (ERV) envelope genes in human endometrial carcinoma and prestages: Emergence of new molecular targets. Oncotarget, 2012. 3(10): p. 1204.

27. Babaian, A., et al., Onco-exaptation of an endogenous retroviral LTR drives IRF5 expression in Hodgkin lymphoma. Oncogene, 2016. 35(19): p. 2542-2546.

28. Grundy, E.E., N. Diab, and K.B. Chiappinelli, Transposable element regulation and expression in cancer. FEBS J, 2021.

29. Sexton, C.E. and M.V. Han, Paired-end mappability of transposable elements in the human genome. Mob DNA, 2019. 10: p. 29.

30. Kim, D., B. Langmead, and S.L. Salzberg, HISAT: a fast spliced aligner with low memory requirements. Nature methods, 2015. 12(4): p. 357-360.

31. Liao, Y., G.K. Smyth, and W. Shi, featureCounts: an efficient general purpose program for assigning sequence reads to genomic features. Bioinformatics, 2014. 30(7): p. 923-930.

32. Gentleman, R.C., et al., Bioconductor: open software development for computational biology and bioinformatics. Genome biology, 2004. 5(10): p. R80.

33. Delaneau, O., et al., A complete tool set for molecular QTL discovery and analysis. Nature Communications, 2017. 8.

34. Love, M.I., W. Huber, and S. Anders, Moderated estimation of fold change and dispersion for RNA-seq data with DESeq2. Genome biology, 2014. 15(12): p. 1-21.

35. Storey, J.D. and R. Tibshirani, Statistical significance for genomewide studies. Proc Natl Acad Sci U S A, 2003. 100(16): p. 9440-5.

36. Scutari, M., Learning Bayesian Networks with the bnlearn R Package. 2010, 2010. 35(3): p. 22.

37. Pertea, M., et al., StringTie enables improved reconstruction of a transcriptome from RNA-seq reads. Nature biotechnology, 2015. 33(3): p. 290-295.

38. Quinlan, A.R. and I.M. Hall, BEDTools: a flexible suite of utilities for comparing genomic features. Bioinformatics, 2010. 26(6): p. 841-2. 
606

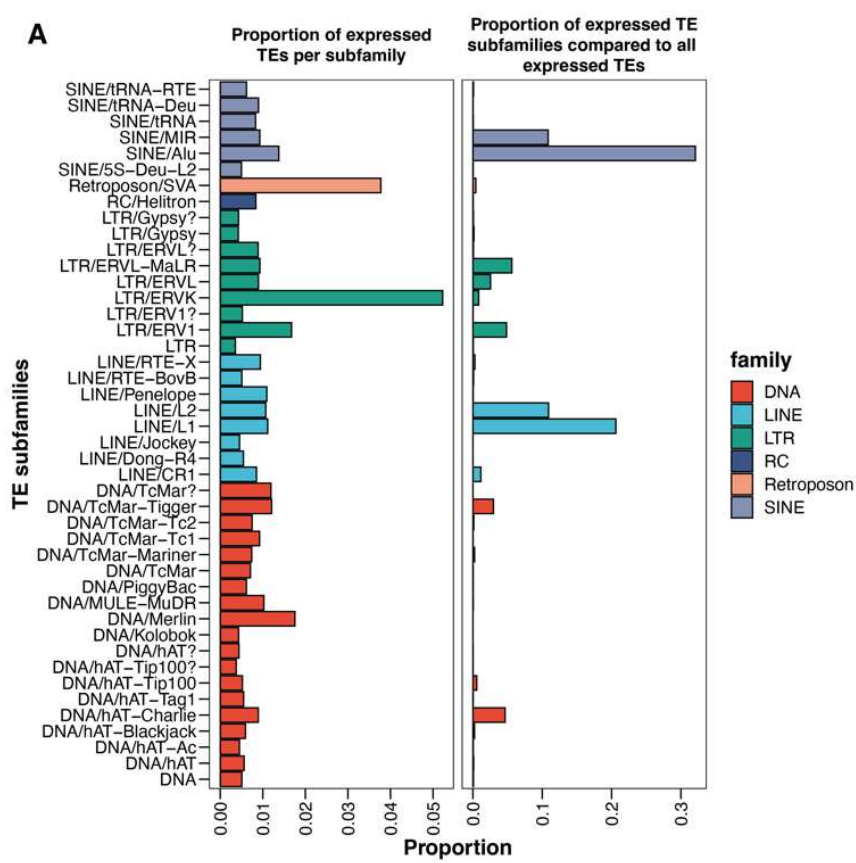

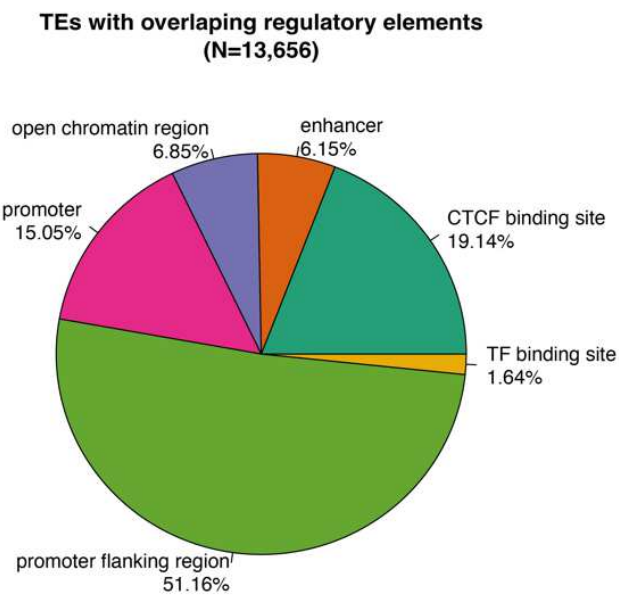

608 Figure 1: Description of quantified TEs. (A) Barplot showing the proportion of uniquely

609 mapped and quantified TE subfamilies in our dataset. (B) Pie chart showing the proportion of

610 TEs with different types of regulatory elements within their sequence. We uniquely mapped

611 and quantified 51,320 TEs. The majority of them are SINEs from the Alu and MIR family, L1

612 and L2 TEs from the LINE family and different subfamilies of LTRs as well as some DNA

613 transposons. When we looked at the proportion of expressed TE per subfamily, we observed

614 that SVA and ERVK are most prominent. Additionally, 13,656 out of the 51,320 TEs contain

615 regulatory elements within sequence. 

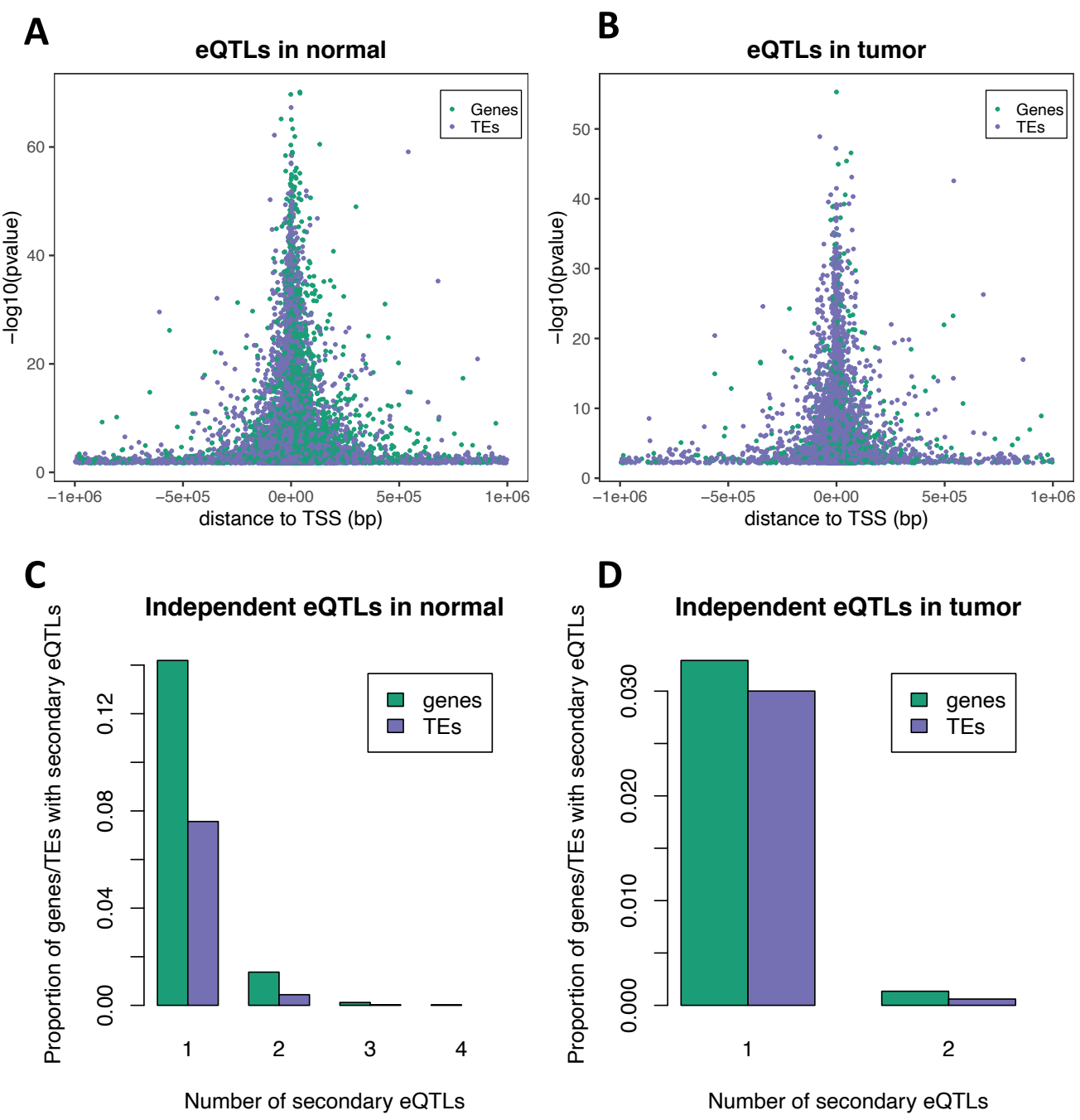

616

617 Figure 2: cis- eQTL discovery. eQTL variant distance to TSS in (A) normal and (B) in tumor.

618 We observe stronger eQTL effect close to the transcription start site of TE and genes in both

619 normal and tumor. Number of secondary eQTLs for TEs and genes in (C) normal and (D)

620 tumor. Gene eQTLs have more functionally independent eQTLs per gene than TEs do. 

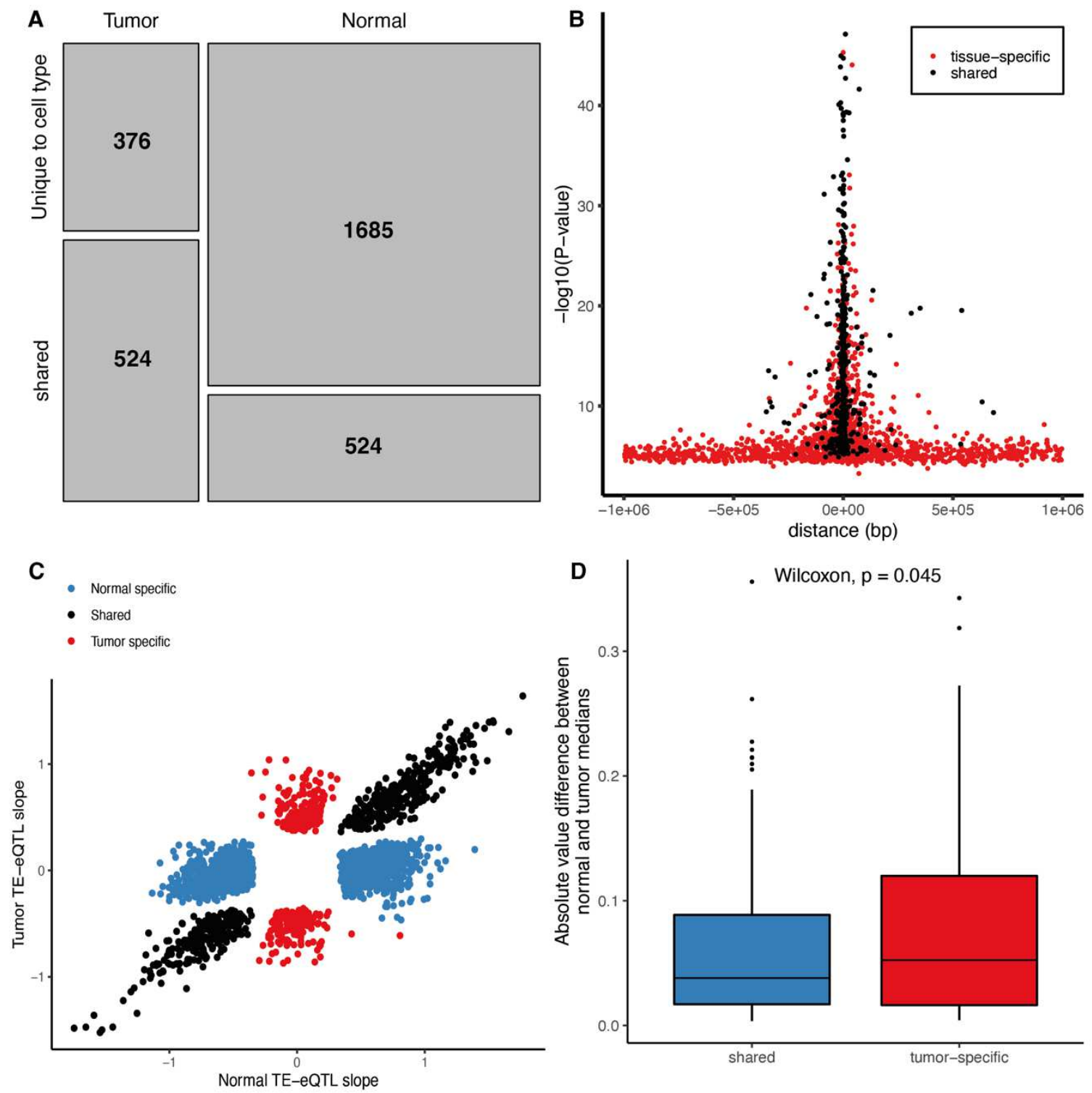

622 Figure 3: Tissue specificity of TE-eQTLs. (A) Mosaic plot of tissue specificity of TE-eQTLs.

623 (B) Tissue specificity and distance of TE-eQTL to transcription start site (TSS). The shared

624 TE-eQTLs (black) are closer to the TSS than are the tissue specific TE-eQTLs (red) (Wilcoxon

$625 \mathrm{P}<2.2 \mathrm{e}-16)$. (C) TE-eQTL slopes for the normal specific TE-eQTLs in blue, the tumor specific 626 in red and shared in black. (D) Boxplot of the absolute value difference of median methylation 627 betas between normal and tumor samples for shared and tumor=specific TE-eQTLs. 


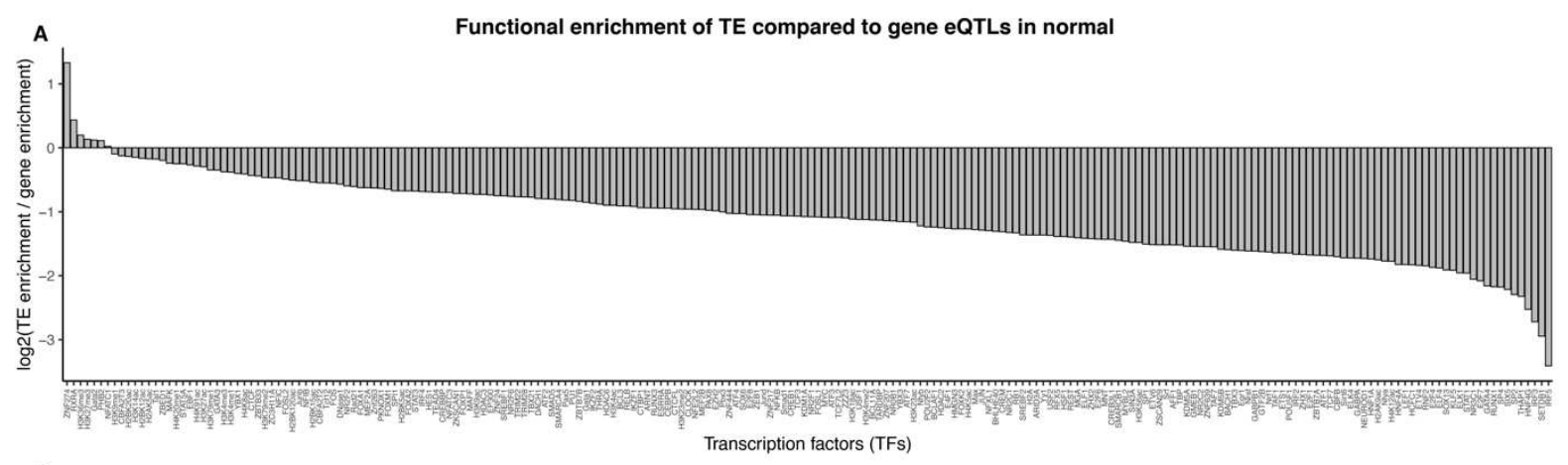

B

Functional enrichment of TE compared to gene eQTLs in tumor

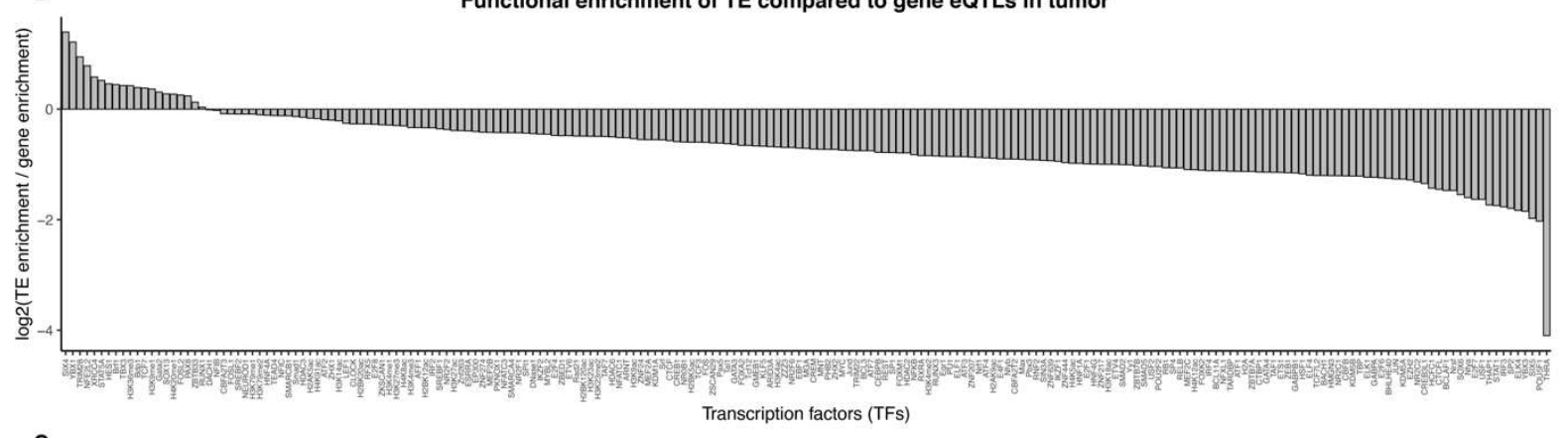

Functional enrichment of tumor-specific compared to shared TE-eQTLs

631 Figure 4 : Functional enrichment of eQTLs. (A) The ratio between TE-eQTL enrichment 632 and gene-eQTL enrichment in log2 scale discovered in normal. 5 TFs show stronger 633 enrichment for TE-eQTLs in normal compared to gene-eQTLs. (B) The ratio between TE634 eQTL enrichment and gene-eQTL enrichment in log2 scale discovered in tumor. We observed 63515 TFs to have a stronger enrichment for TE-eQTLs than gene-eQTLs in normal. (C) log2 ratio 636 between tumor-specific TE-eQTL enrichment and shared TE eQTL enrichment. We observe $63780 \mathrm{TFs}$ with a stronger enrichment for the tumor-specific TE-eQTLs than the shared eQTLs 638 indicating that these TFs regulate TE expression specifically in tumor. 
A Genome-wide mean probability

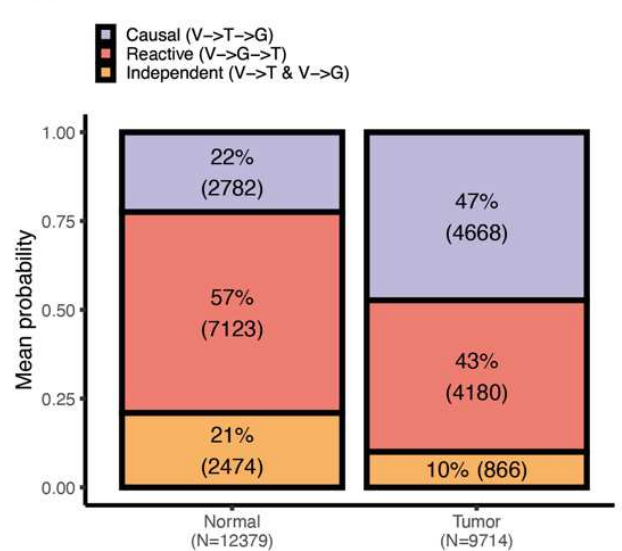

C

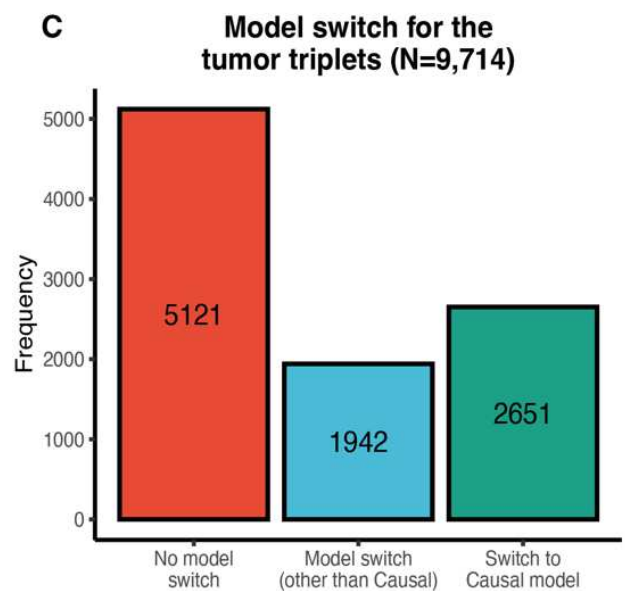

B Model substitutions for the significant tumor triplets $(\mathrm{N}=9714)$
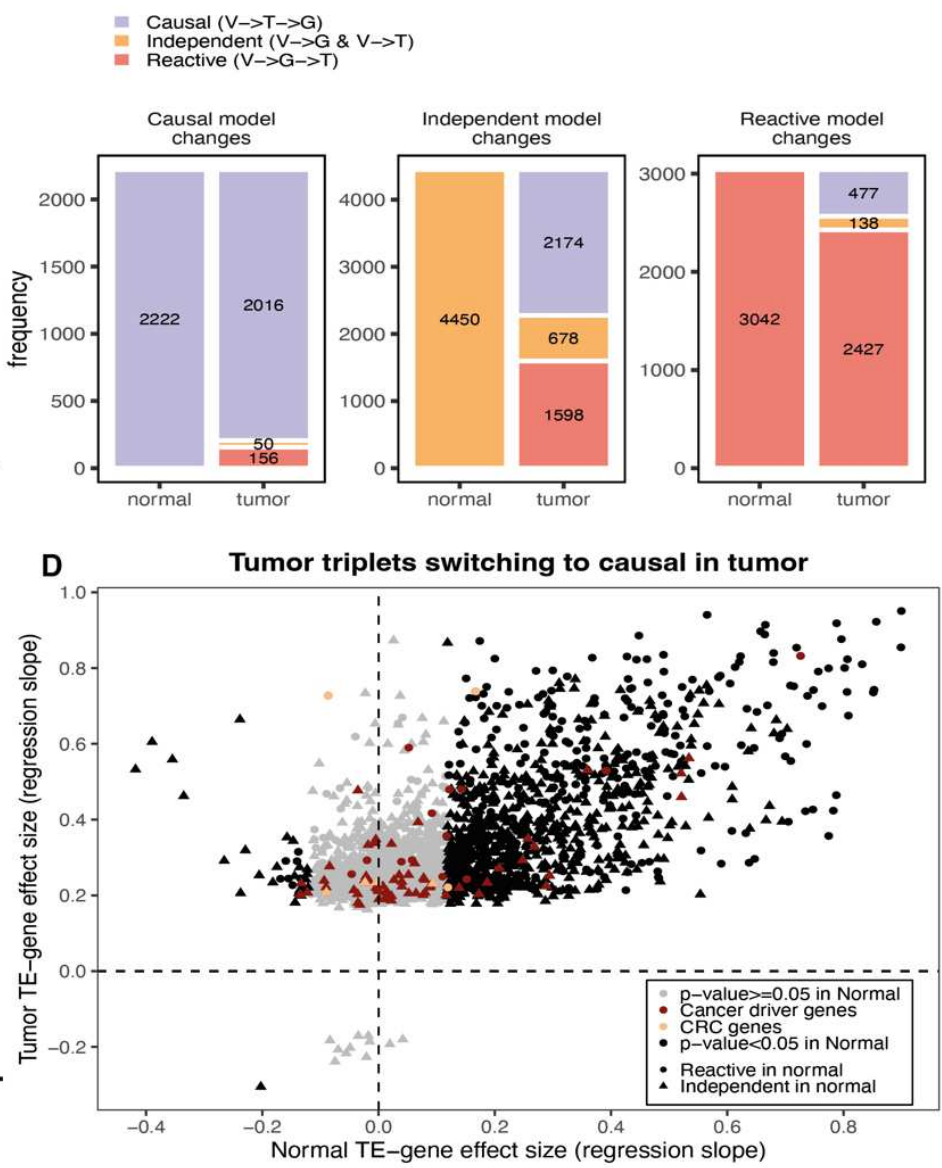

Figure 5: Causal relationship between eQTL variants, TEs and genes. (A) Barplot

642 representing the mean probability for each of the three models in normal and tumor. We

643 observe significantly more causal cases in tumor compared to normal (Wilcox P-value $<2 e-$

644 16) (B) Barplot representing the model substitutions for the 9,714 tumor triplets from normal

645 to tumor. Independent models tend to shift to a causal in tumor. This is true also for the reactive

646 models in normal but to a much smaller extent. (C) Barplot representing the number of triplets

647 that do not switch models, that switch to a causal model or that switch to reactive/independent

648 from normal to tumor. The majority of triplets do not switch models between normal and tumor.

649 However, 2,651 triplets are switching to a causal model making the corresponding TEs

650 potential drivers of gene expression

651 (D) Each point represents a TE-gene for each of the 2,561 tumor triplets. All points aresignificant in tumor but not in normal (grey points). We observe that in most cases, TEs 
653 are positively correlated with genes except for a few cases. Most cancer driver genes have no 654 significant correlation with any TE in normal indicating that for most part, TEs impact them 655 specifically in tumor.

656

657
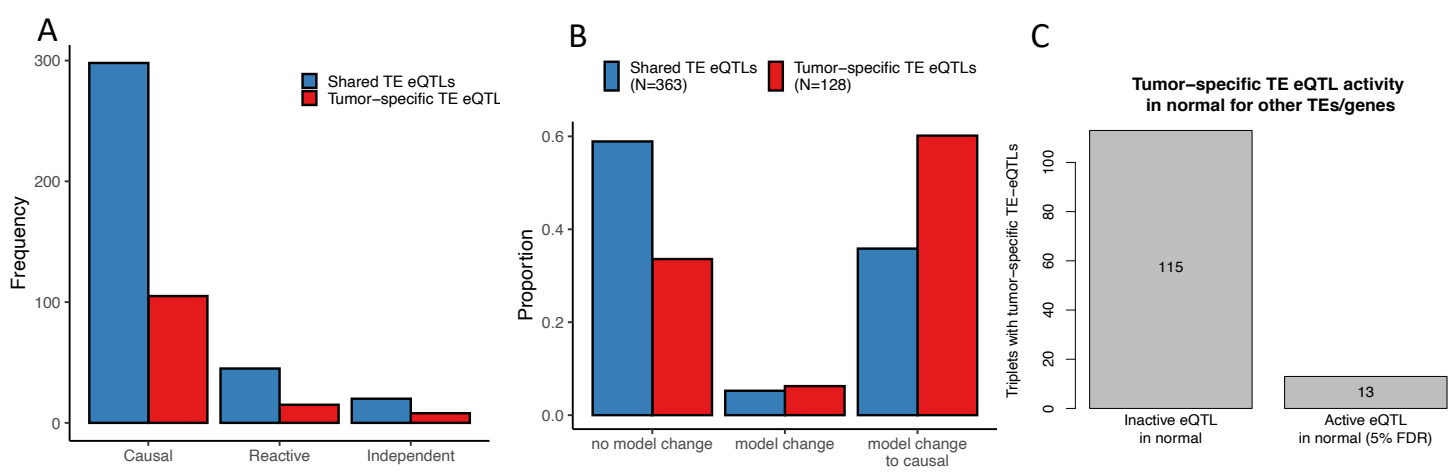

658

659 Figure 6: Tumor-specific and shared TE-eQTLs effects. (A) The barplot represent the 660 frequency of the causal, reactive and independent model for the triplets with shared or tumor661 specific TE-eQTLs (B) the barplot represents the model changes from normal to tumor for the 662 triplets constituted of shared or tumor-specific TE-eQTLs. (C) represent the effect size of the 663 shared and tumor-specific TE-eQTLs. (D) Barplot that represent the number of tumor-specific 664 TE-eQTLs that are inactive eQTLs for the triplet associated gene.

665 


\section{Supplementary information}

\section{Transposable elements mediate genetic effects altering the expression of nearby genes in colorectal cancer}

Nikolaos M. R. Lykoskoufis ${ }^{1,2,3^{*}}$, Evarist Planet ${ }^{4}$, Halit Ongen ${ }^{1,2,3}$, Didier Trono ${ }^{4}$, Emmanouil T. Dermitzakis $^{1,2,3^{*}}$

${ }^{1}$ Department of Genetic Medicine and Development, University of Geneva Medical School, 1211 Geneva, Switzerland

${ }^{2}$ Institute for Genetics and Genomics in Geneva (iGE3), University of Geneva, 1211 Geneva, Switzerland

${ }^{3}$ Swiss Institute of Bioinformatics, 1211 Geneva, Switzerland

${ }^{4}$ School of Life Sciences, Ecole Polytechnique Fédérale de Lausanne (EPFL), 1015, Lausanne, Switzerland

* Corresponding authors: N.M.R Lykoskoufis: nikolaos.lykoskoufis@unige.ch and E.T.D: emmanouil.dermitzakis@unige.ch

Department of Genetic Medicine and Development, University of Geneva,

1 rue Michel-Servet, 1211 Geneva, Switzerland 


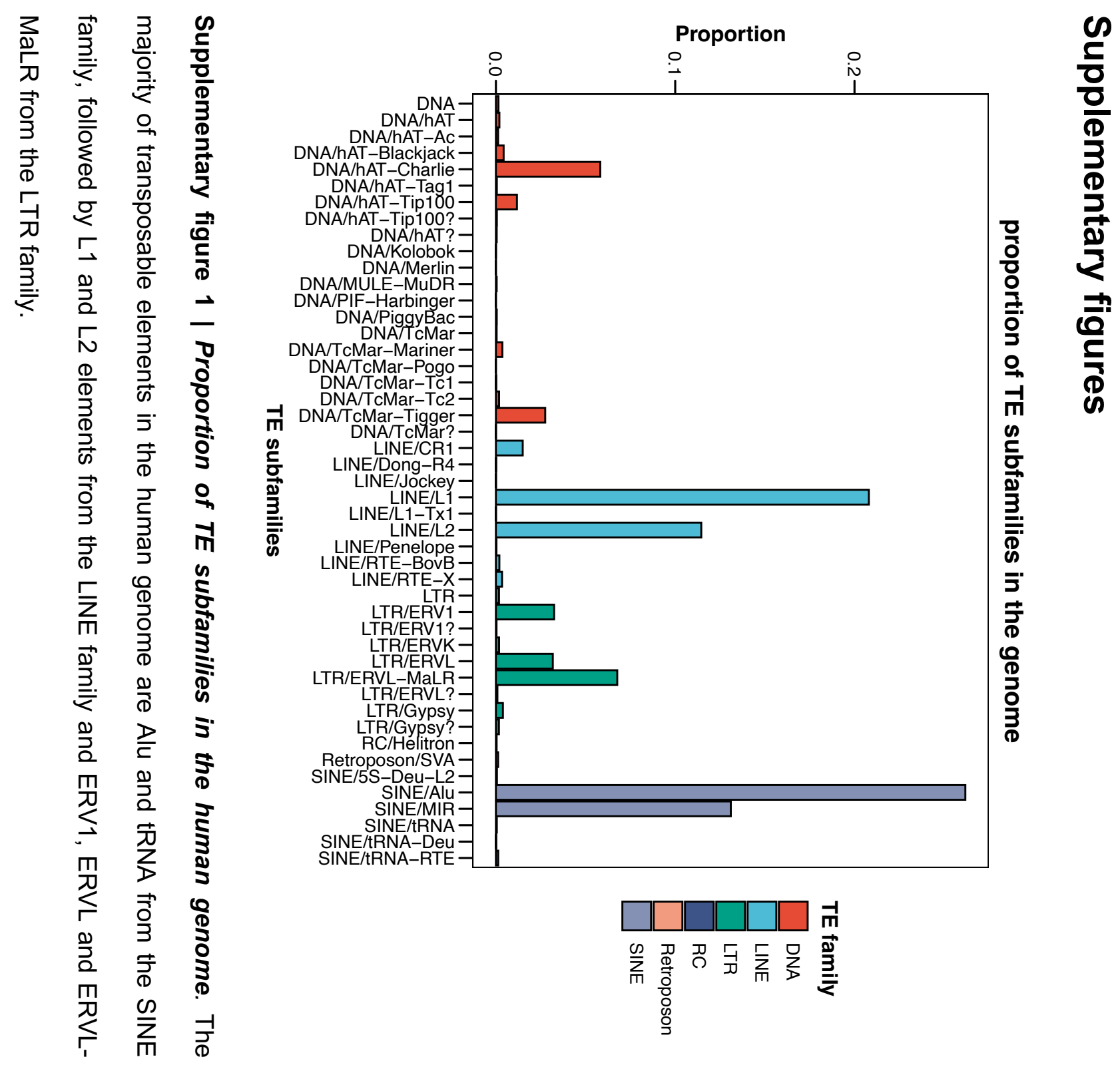




\section{TEs with overlapping regulatory regions in the genome}

$(\mathrm{N}=820,981)$

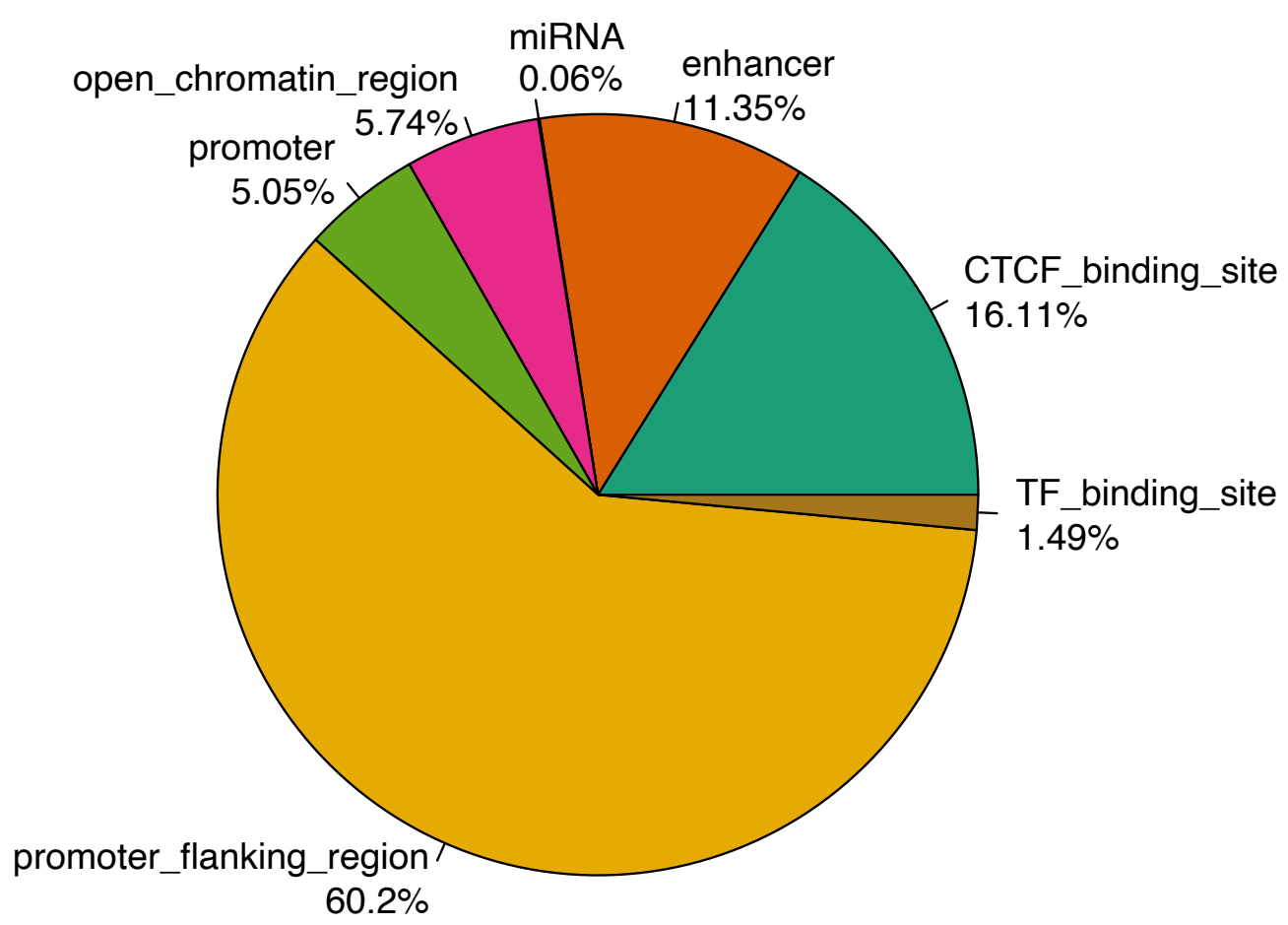

Supplementary Figure 2 | TEs overlapping regulatory regions in the human genome.

Pie plot representing the proportion of transposable elements overlapping with each regulatory regions. Of the $\sim 4.6$ million TEs in the human genome, 820,981 TEs are overlapping with at least one regulatory region. 
A. normal eQTL association signal

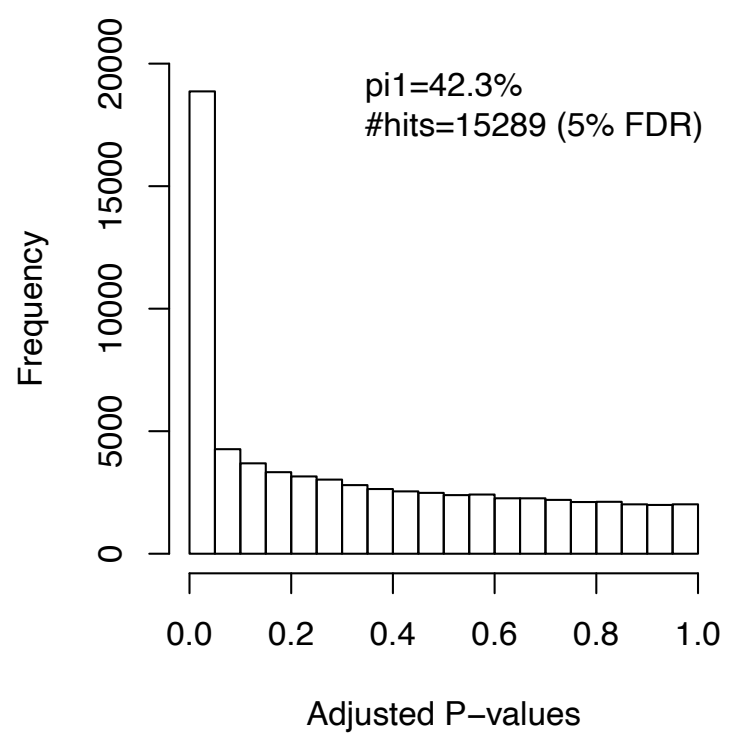

B. tumor eQTL association signal

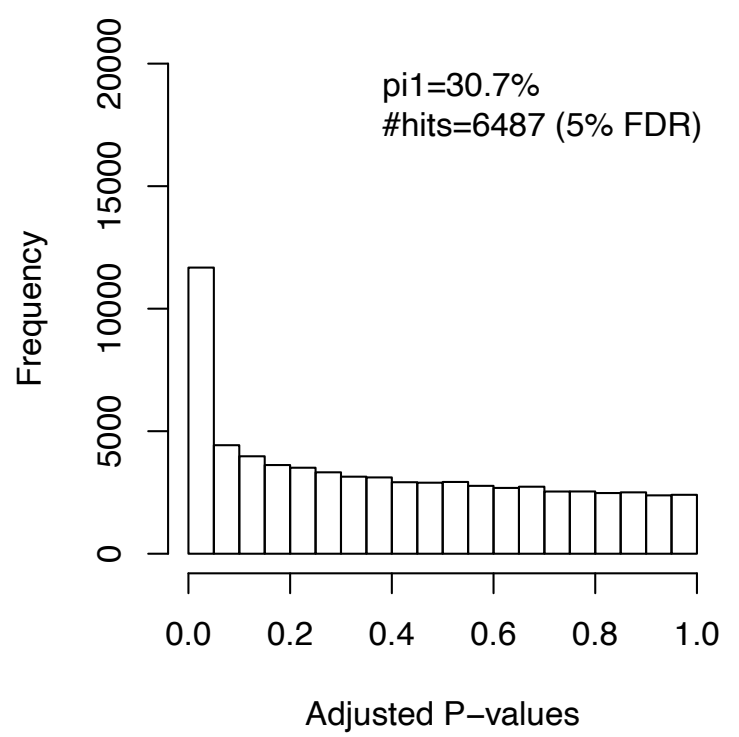

Supplementary figure 3 | cis eQTL discovery p-value distribution. Histograms of $p$-value distribution of cis- eQTL discovery in (A) normal and (B) tumor. 


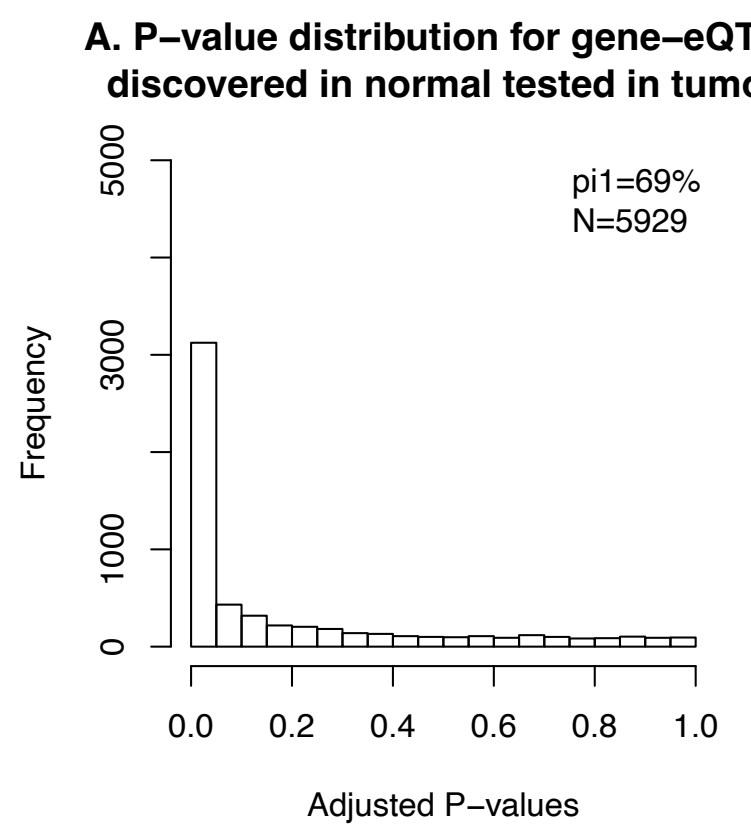

A. P-value distribution for TE-eQTLs discovered in normal tested in tumor

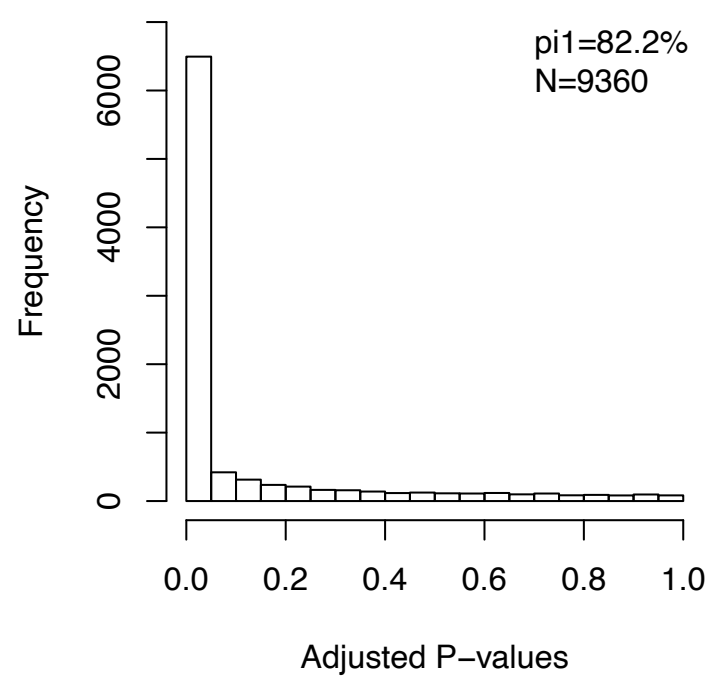

B. P-value distribution for gene-eQTLs discovered in tumor tested in normal

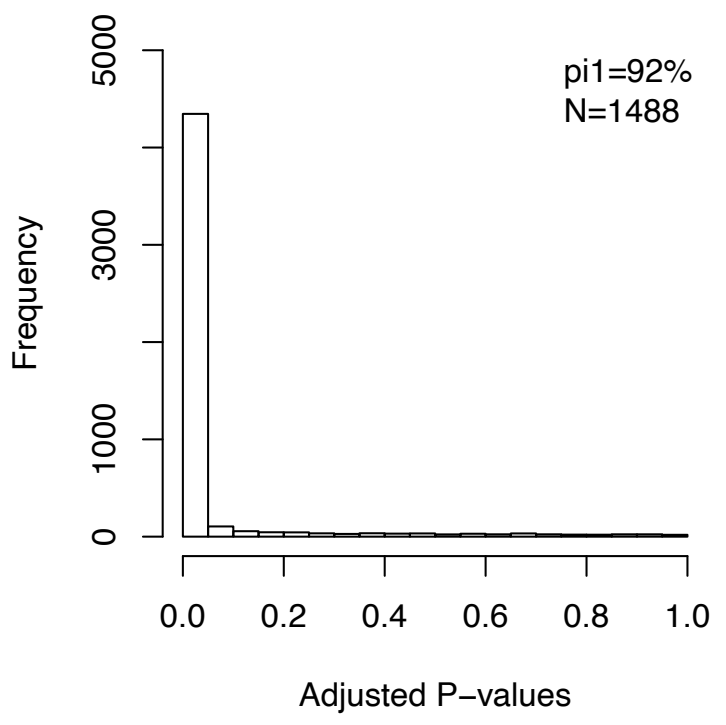

B. P-value distribution for TE-eQTLs discovered in tumor tested in normal

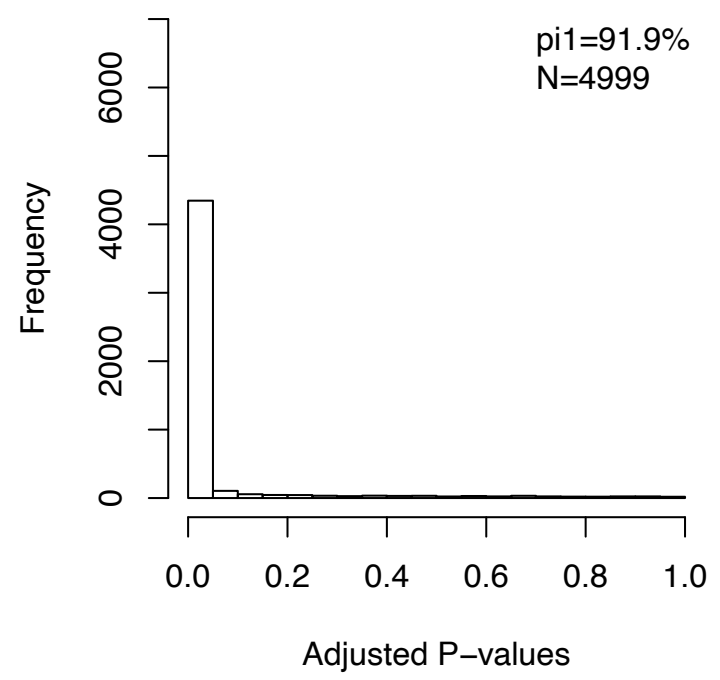

Supplementary figure 4 | P-value distributions of significant SNP-gene or SNP-TE pairs tested in the other tissue. The $\pi_{1}$ statistic estimates the tissue sharing of eQTLs. We observe that SNP-TE and SNP-gene pairs discovered in tumor are replicated better in normal than normal eQTLs tested in tumor. 
A

Normal

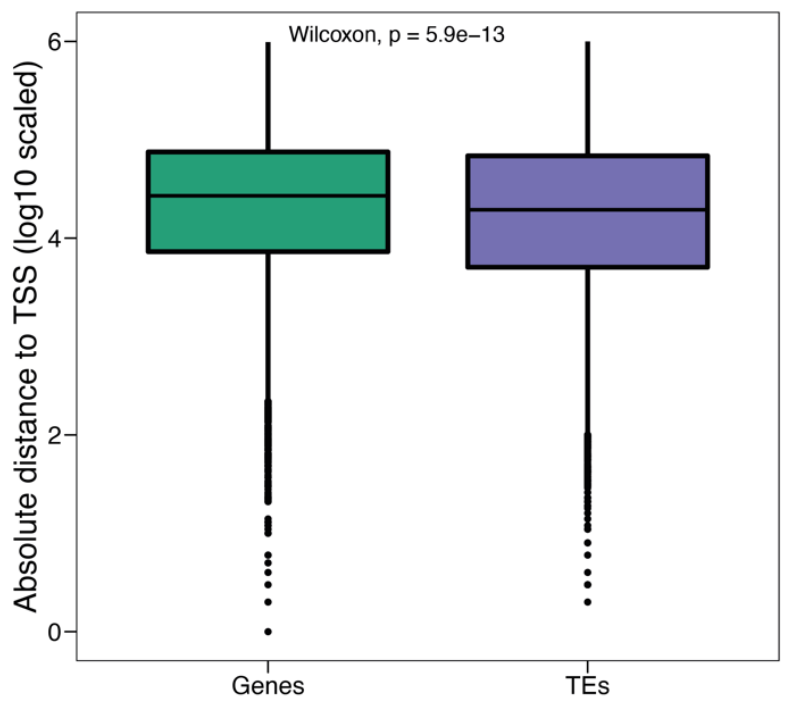

B

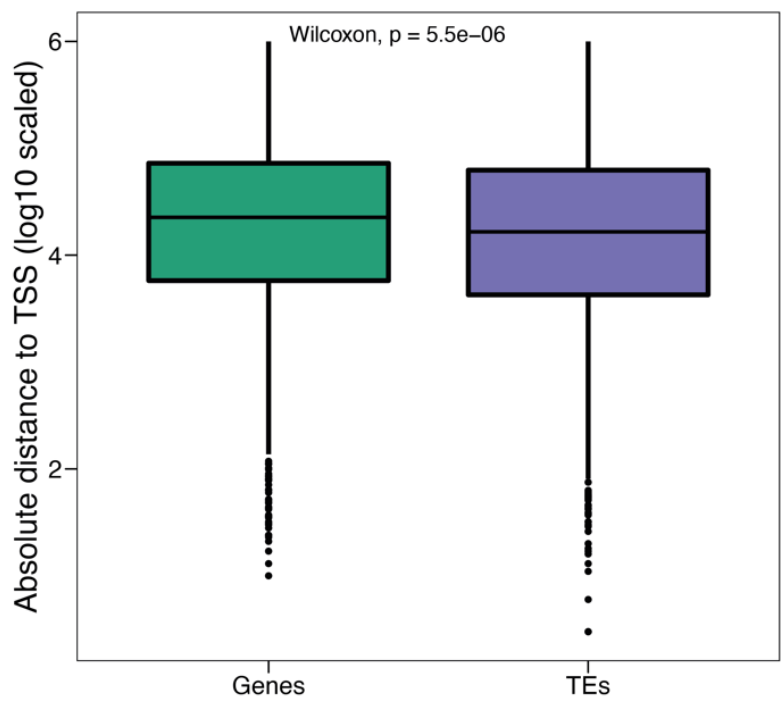

Supplementary figure 5 | TE and gene eQTL distance to TSS in normal and tumor. We observe that in both normal and tumor, TE-eQTLs are closer to the TSS of TEs than gene-eQTLs are to the TSS of genes. This could be because of the smaller evolutionary time TEs have in the human genome compared to genes, making local effects much more likely to occur. 


\section{eQTL allele frequencies}

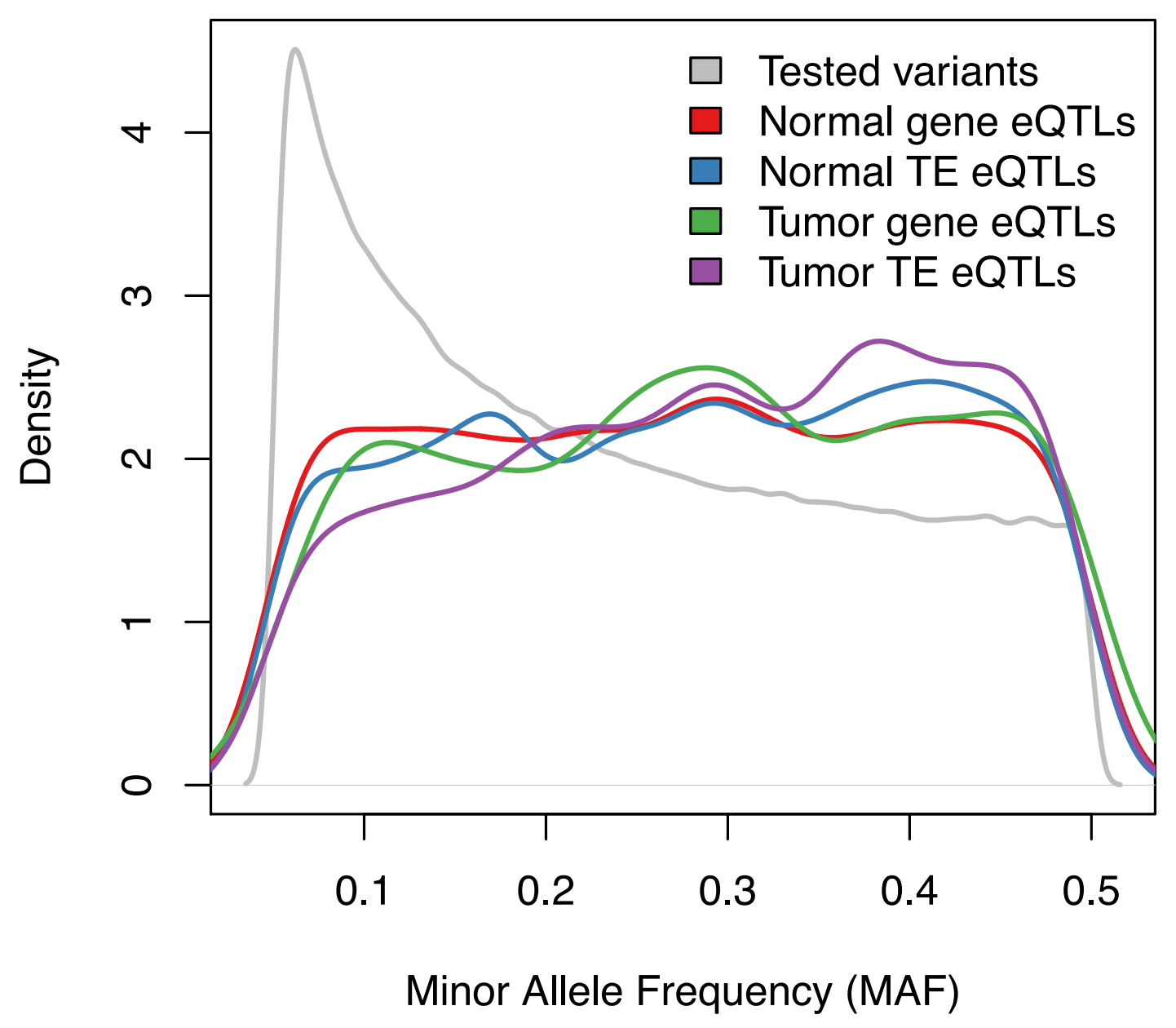

Supplementary figure 6 | eQTL variant allele frequencies. We observe that the allele frequencies for the TE- and gene-eQTLs are very similar in normal and tumor. 
A

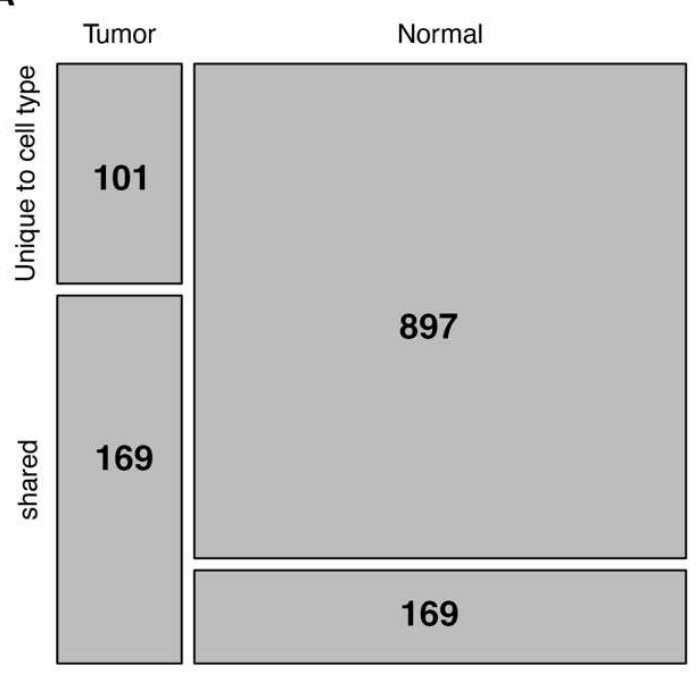

C

- Shared

- Tumor specific

- Normal specific

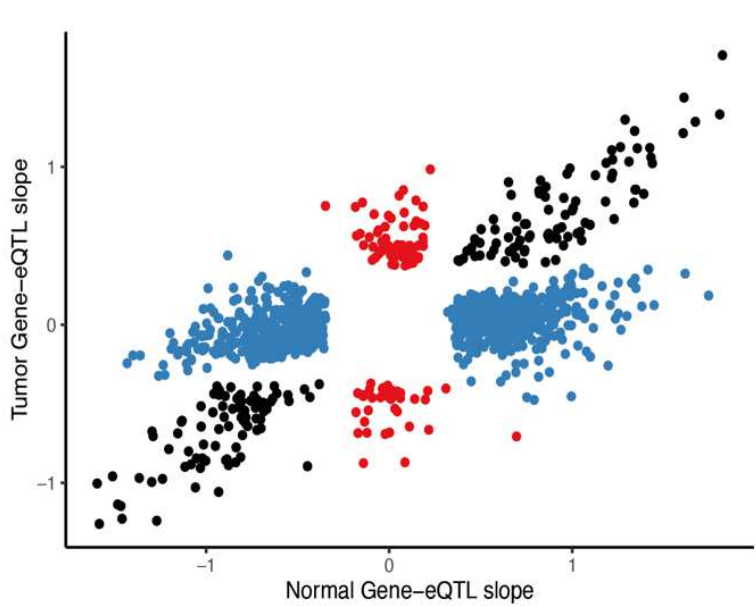

B

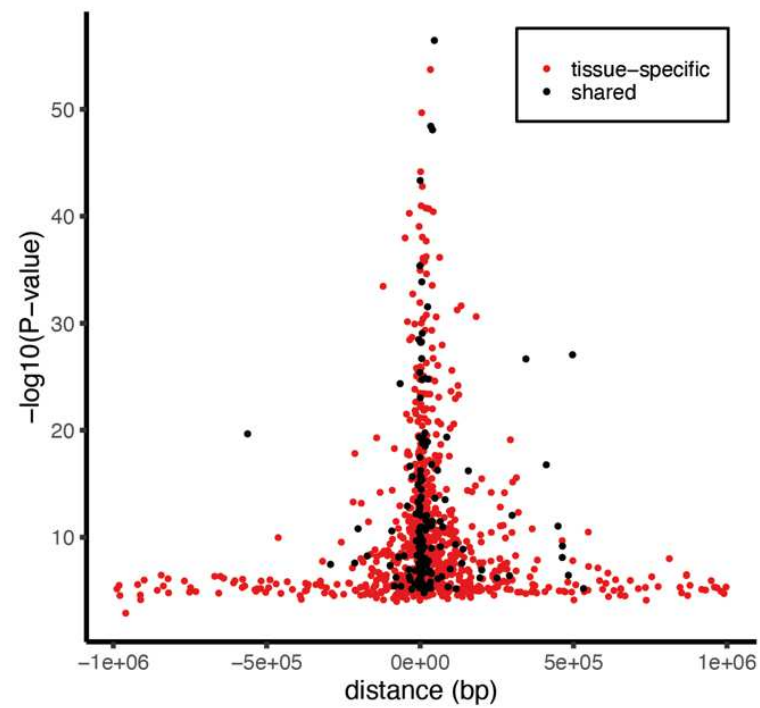

D ${ }^{0.25}$ - Wilcoxon, $p=0.0055$

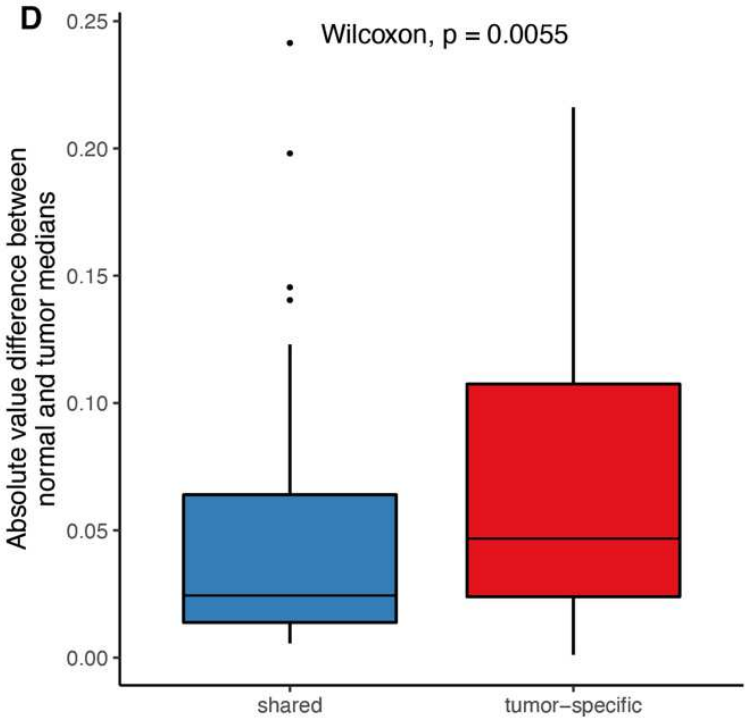

Supplementary figure 7 | Tissue specificity of Gene-eQTLs. (A) Mosaic plot of tissue specificity of Gene-eQTLs. (B) Tissue specificity and distance of Gene-eQTL to transcription start site (TSS). The shared Gene-eQTLs (black) are closer to the TSS than are the tissue specific Gene-eQTLs (red) (Wilcoxon P<2.2e-16). (C) Gene-eQTL slopes for the normal specific Gene-eQTLs in blue, the tumor specific in red and shared in black. (D) Boxplot of the absolute value difference of median methylation betas between normal and tumor samples for shared and tumor-specific gene-eQTLs. 


\section{Functional enrichment for normal gene and TE eQTLs}

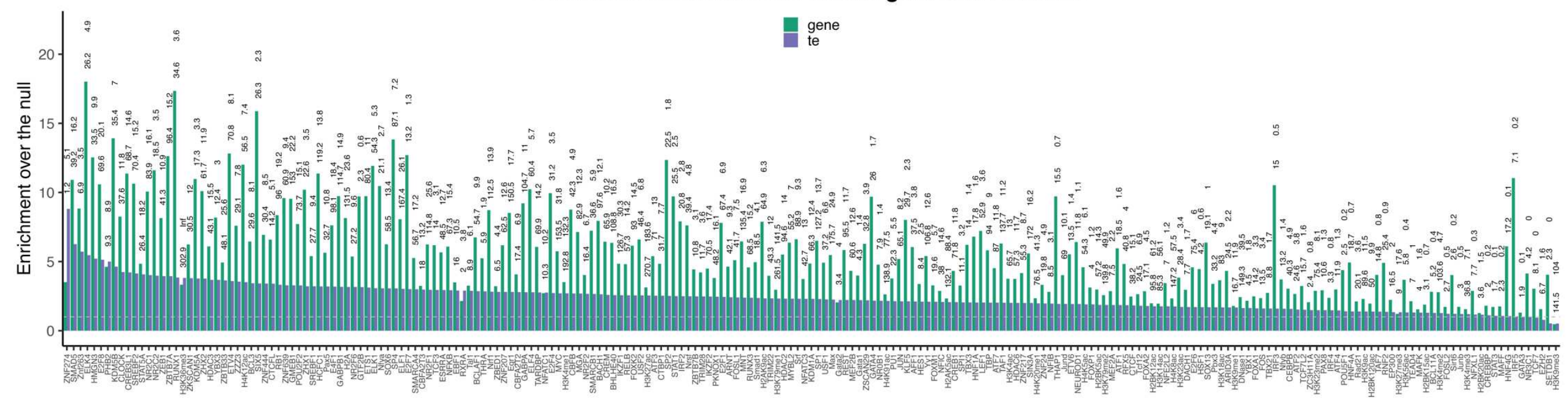

Supplementary Figure 8 | Functional enrichment for gene- and TE-eQTLs in normal. Enrichment over the null for gene- and TE-eQTLs in normal. Only plotting cases where the enrichment was significant at 5\% FDR for either gene- or TE-eQTLs. 


\section{Functional enrichment for tumor gene and TE eQTLs}

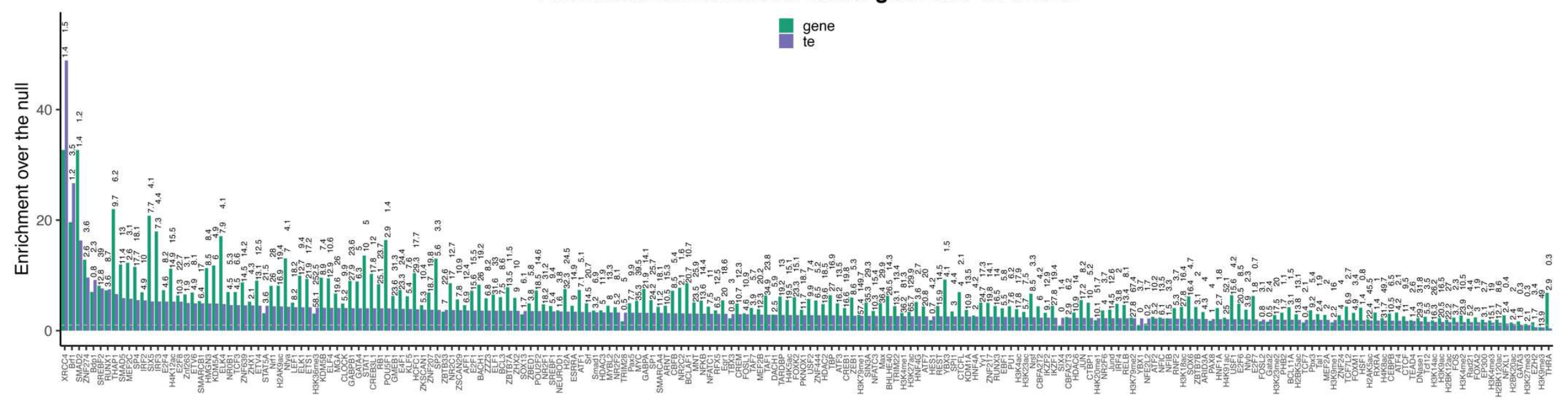

Supplementary Figure 9 | Functional enrichment for gene- and TE-eQTLs in tumor. Enrichment over the null for gene- and TE-eQTLs in tumor. Only plotting cases where the enrichment was significant at 5\% FDR for either gene- or TE-eQTLs. 


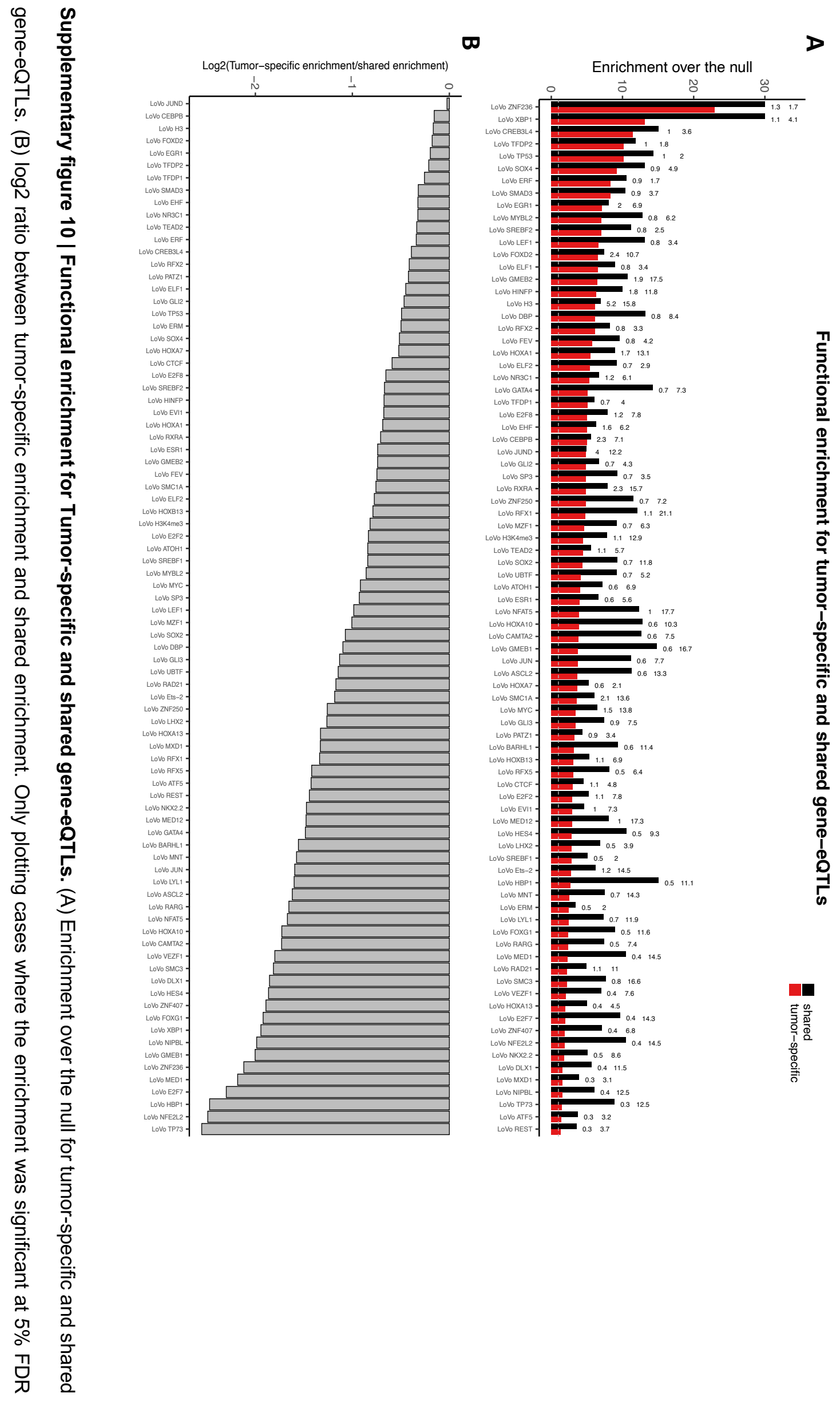


for either tumor-specific or shared gene-eQTLs. None of the TFs have a stronger enrichment for tumor-specific gene-eQTLs indicating that these TFs regulate gene expression in both the normal and tumor state.

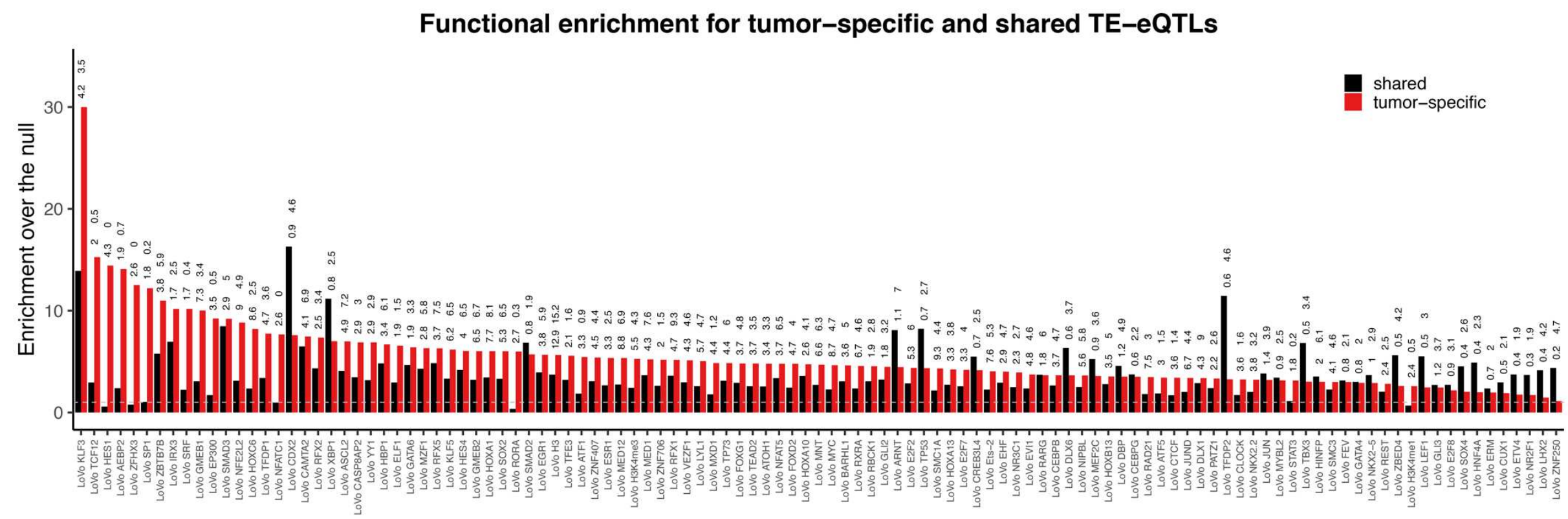

Supplementary figure 11 | Functional enrichment for Tumor-specific and shared TE-eQTLs. (A) Enrichment over the null for tumor-specific and shared TE-eQTLs. Only plotting cases where the enrichment was significant at 5\% FDR for either tumor-specific or shared TE-eQTLs. 


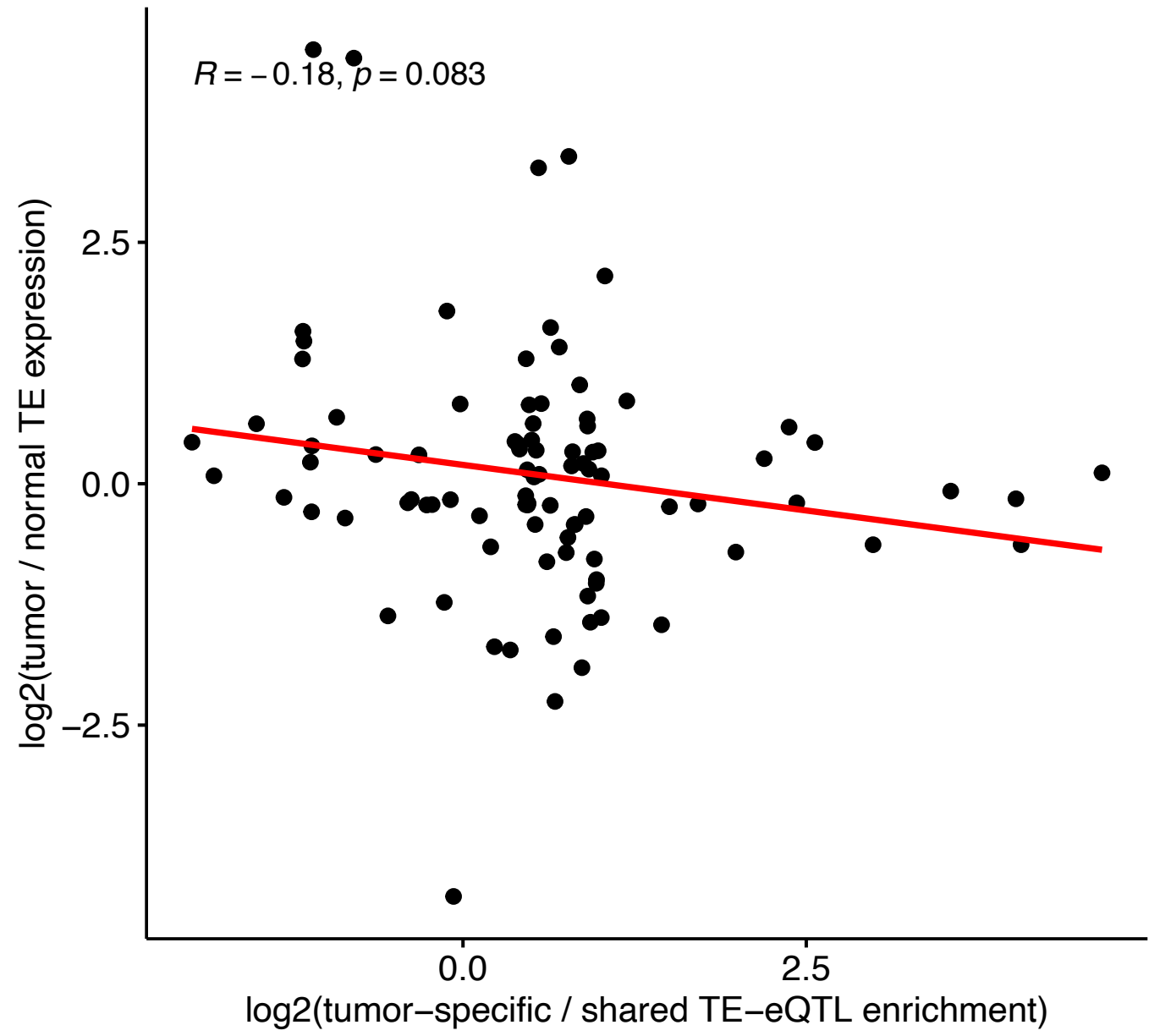

Supplementary figure 12 | Correlation of differential enrichment of functional binding sites and differential expression of the corresponding transcription factors. We compared the ratio of tumor expression over the normal expression of differentially expressed transcription factors to the ratio of enrichment for the binding sites of the same transcription factors in tumor specific TE-eQTLs over the shared TE-eQTLs. We find no significant correlation indicating that differential expression of the corresponding TFs do not drive the tumor-specific TE-eQTLs. 


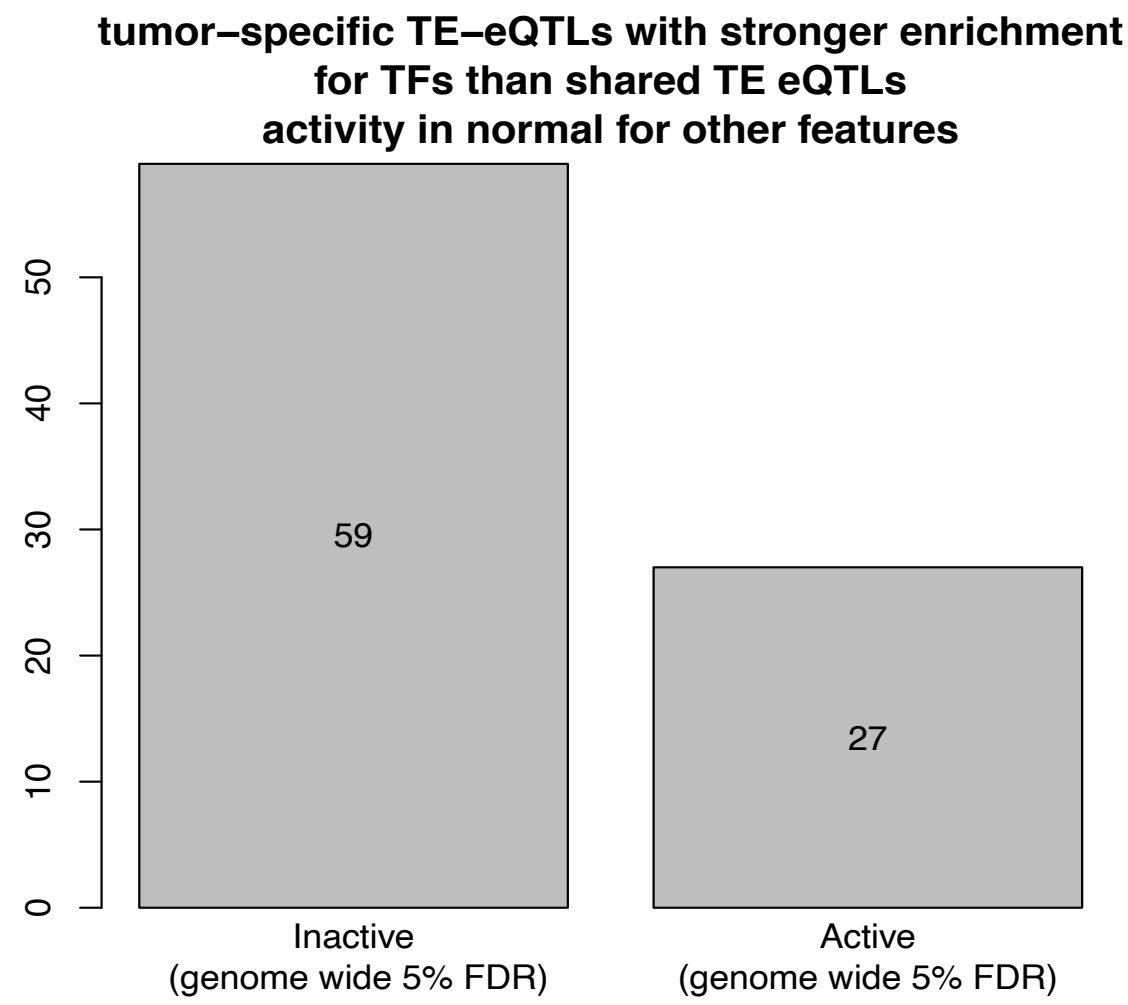

Supplementary figure 13 | Tumor-specific TE-eQTLs overlapping with the enriched transcription factors and their activity in normal regarding other genes/TEs. We checked whether any of the tumor-specific TE-eQTLs overlapping with the transcription factors we found to have a stronger enrichment for tumor-specific TE-eQTLs are active or inactive eQTLs for other TEs or genes in cis. We discovered that 59 of them are not significant eQTLs for any TE or gene in normal indicating that these regions are probably inactive and get activated in tumorigenesis. 
A Number of genes associated per TE in normal

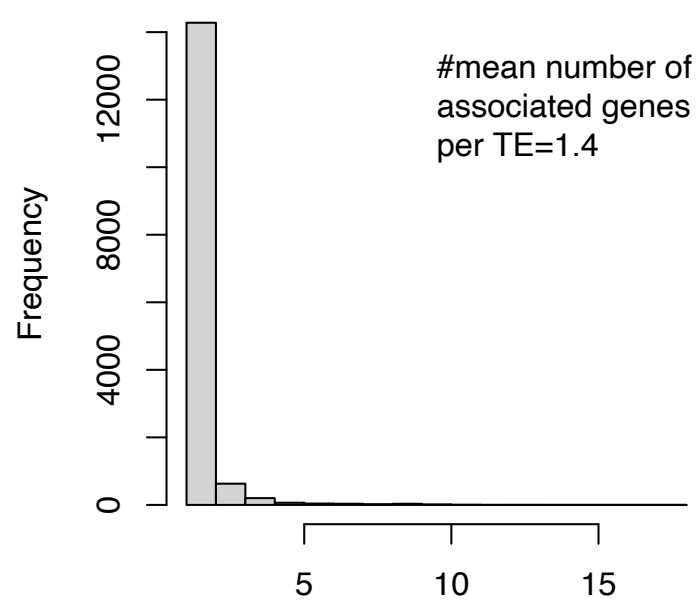

Number of genes associated per TE
B Number of genes associated per TE in tumor

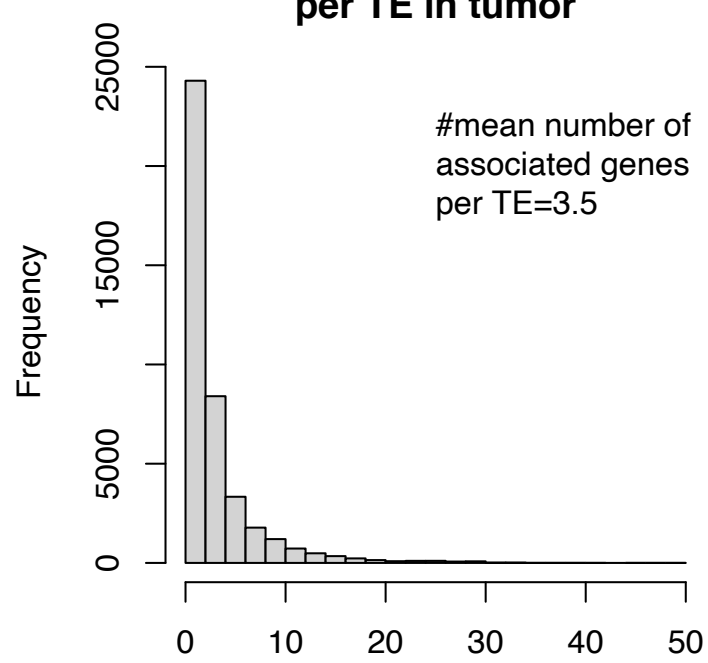

Number of genes associated per TE

Supplementary figure 14 | Mean number of genes associated per TE in (A) normal and (B) tumor.

We observe that in normal there are less genes associated per TE (mean number of associated genes per TE $=1.4$ ) compared to tumor (mean number of associated genes per TE $=3.5$ ) 

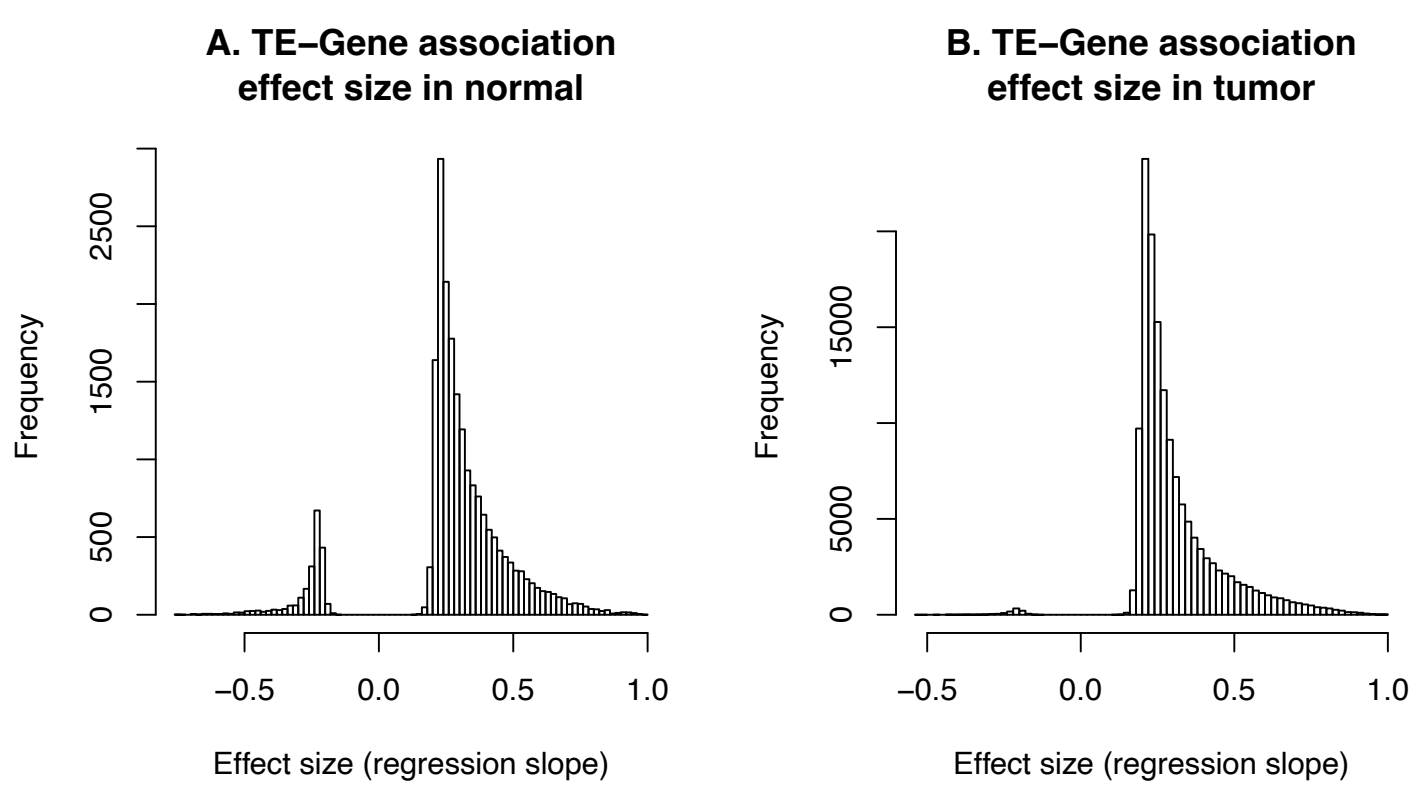

Supplementary figure 15 | TE-gene effect sizes (regression slope) in (A) normal and (B) tumor. We observe that most TEs are positively associated with a gene. 
A. SNP-TE-gene associations in normal

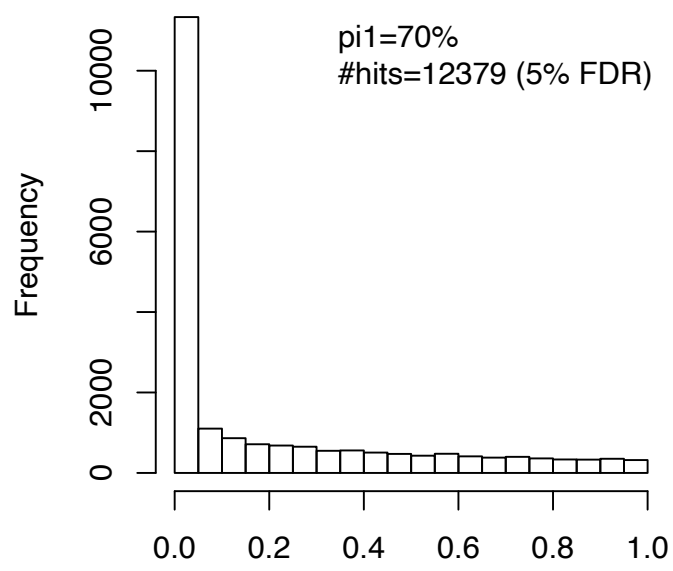

Adjusted P-values
B. SNP-TE-gene associations in tumor

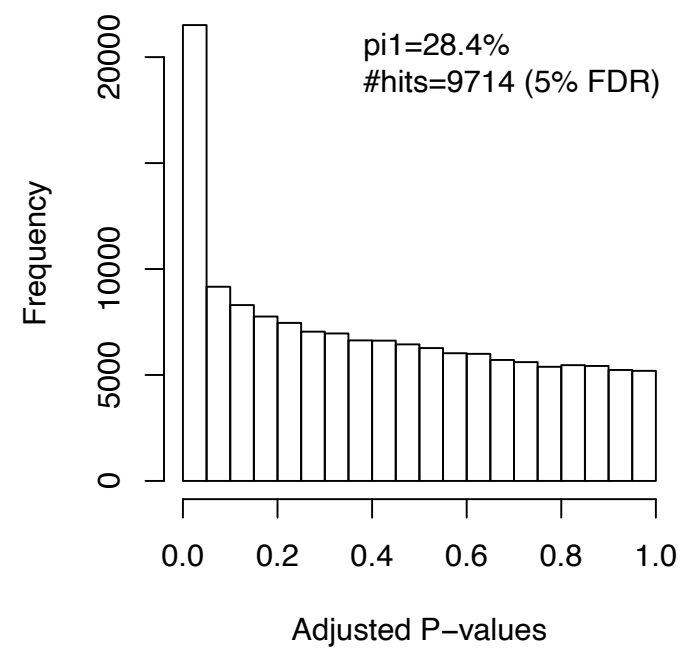

Supplementary figure 16 | P-value distribution of eQTL TE-gene discovery in (A) normal and (B) tumor. We observe that in normal (A) we discovered 12,379 triplets and in (B) tumor 9,714 triplets at $5 \%$ FDR. 


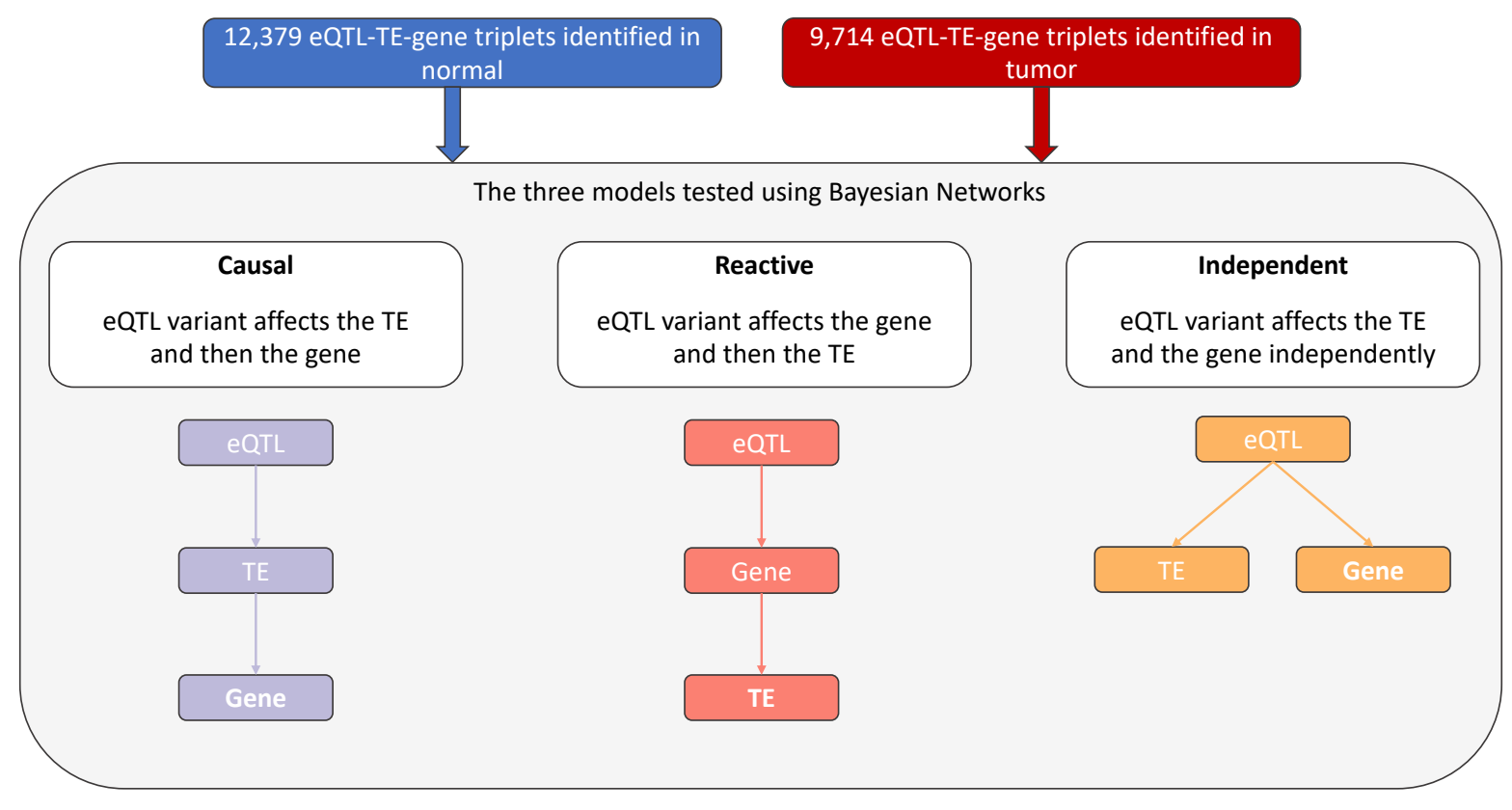

Supplementary figure 17 | Causal relationship of eQTLs, TEs and genes approach. To infer the most likely causal relationship between eQTL variants, TEs and genes, we tested three models using Bayesian Networks (BNs). The causal model where the eQTL variant affects the TE and then the gene, the Reactive model where the eQTL variants affects the gene and then the TE and the Independent model where the eQTL variant affects the TE and gene independently. For each triplet we obtained log likelihoods and we calculated posterior probabilities using a uniform prior probability for each of the three models. 


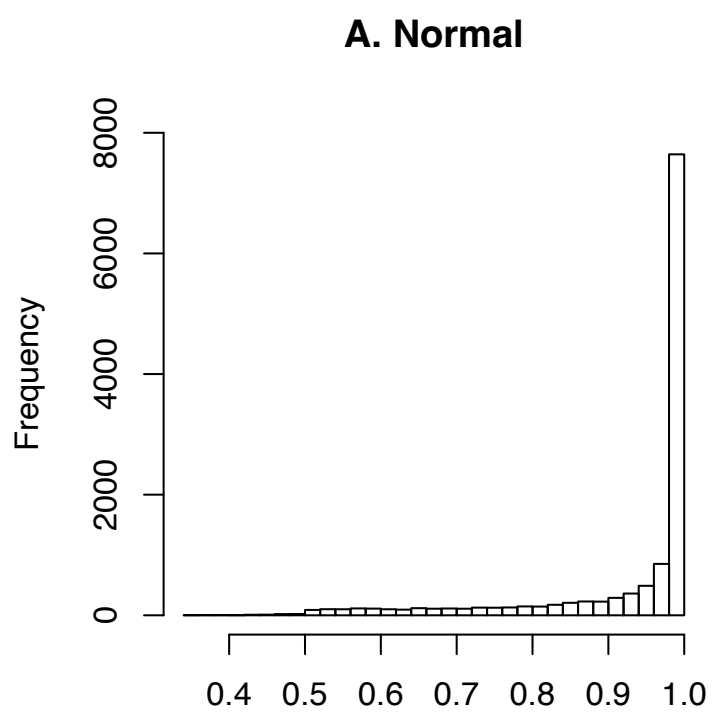

Probability of the most likely model
B. Tumor

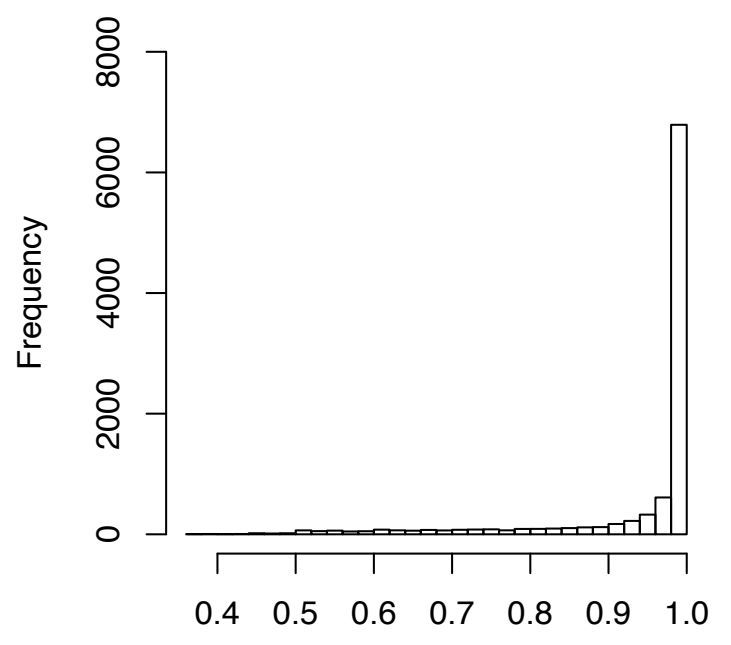

Probability of the most likely model

Supplementary figure 18 | Probability of the most likely model in (A) normal and (B) tumor. We can see that the probabilities of the most likely models are for most part above 0.8 . 
Causal $(V->T->G)$

Reactive $(V \rightarrow-G \rightarrow T)$

Independent $(\mathrm{V} \rightarrow \mathrm{G} \& \mathrm{~V} \rightarrow \mathrm{T})$

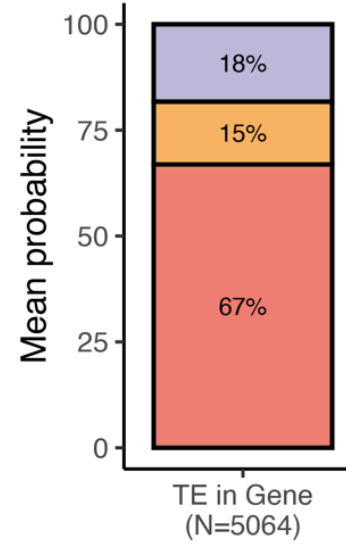

B

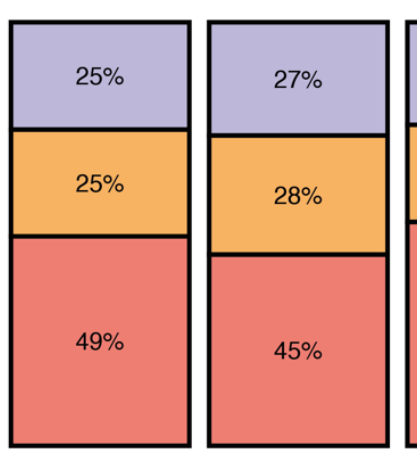

ToutG

\section{TE}

of gene

$(\mathrm{N}=3242)$
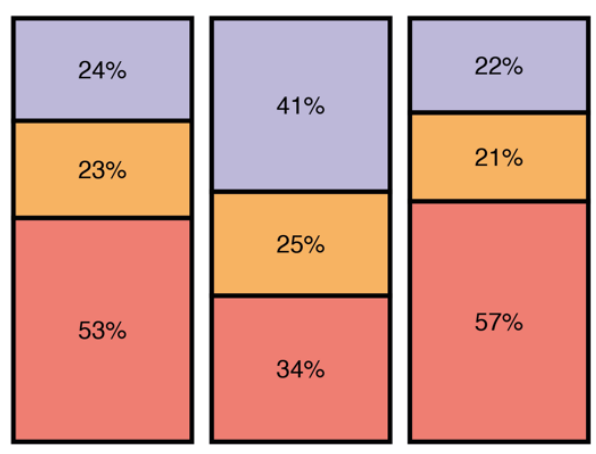

Variant outside

TE

$(\mathrm{N}=12268)$

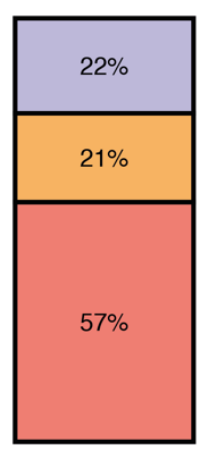

All cases

Together

$(\mathrm{N}=12379)$

Tumor $(\mathrm{N}=9714)$

Causal $(\mathrm{V}->\mathrm{T}->\mathrm{G})$
Reactive $(\mathrm{V} \rightarrow \mathrm{G}->\mathrm{T})$

Independent $(\mathrm{V} \rightarrow \mathrm{G}$ \& $\mathrm{V}->\mathrm{T})$
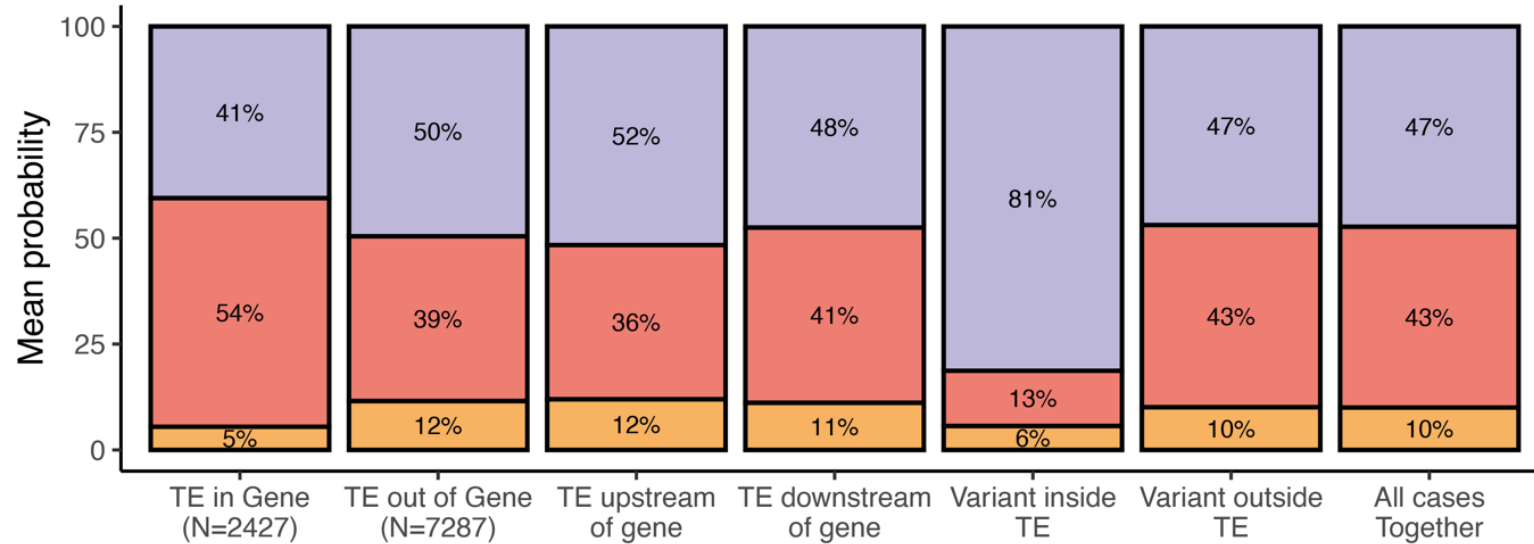

$(\mathrm{N}=2427)$

$(\mathrm{N}=7287)$

TE upstrea
of gene

E downstre
of gene

Varian

TE

$(\mathrm{N}=119)$

Variant outside
TE

All cases

Together

$(\mathrm{N}=3624)$

$(\mathrm{N}=3663)$

$(\mathrm{N}=9595)$

$(\mathrm{N}=9714)$

Supplementary figure 19 | Causal relationships depending on the genomic position of the TE in

respect to the gene. In each case, we worked out the percentages by averaging the posteriors given by the Bayesian networks across all the triplets falling in each of the categories. We observe that TEs inside genes or downstream of genes tend to react to gene expression whereas TEs outside genes or when the eQTL variant is within the TE sequence, TEs are most likely causal for changes in gene expression. 
A Shared triplets $(\mathrm{N}=1592)$ genome-wide mean probability

Causal $(\mathrm{V} \rightarrow \mathrm{T}->\mathrm{G})$

Independent $(\mathrm{V} \rightarrow \mathrm{T} \& \mathrm{~V} \rightarrow \mathrm{G})$

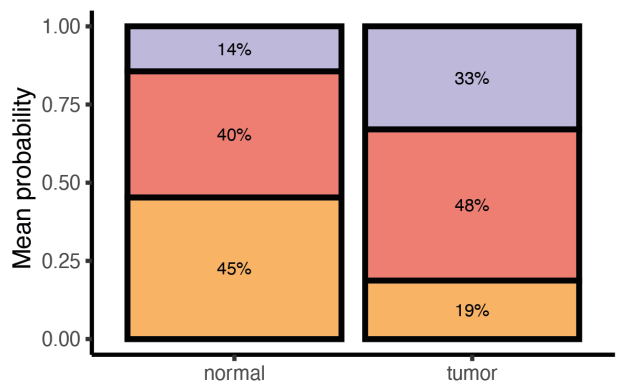

C Union of normal and tumor triplets $(\mathrm{N}=21647)$

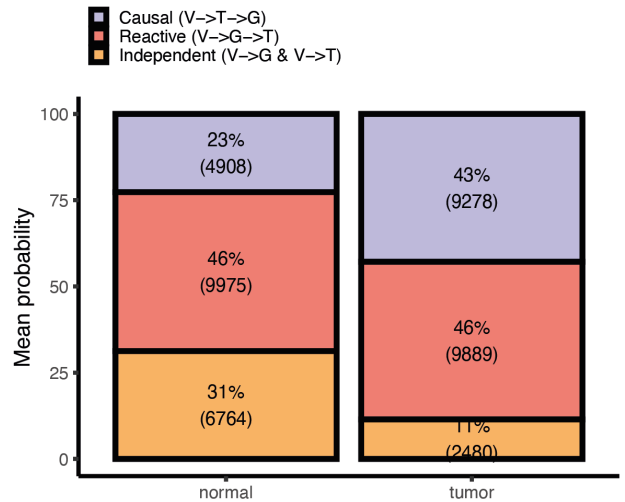

B

Model substitutions between normal and tumor

for shared triplets $(\mathrm{N}=1592)$

Causal $(V \rightarrow T->G)$

Independent $(\mathrm{V} \rightarrow \rightarrow \mathrm{G} \& \mathrm{~V} \rightarrow \mathrm{T})$
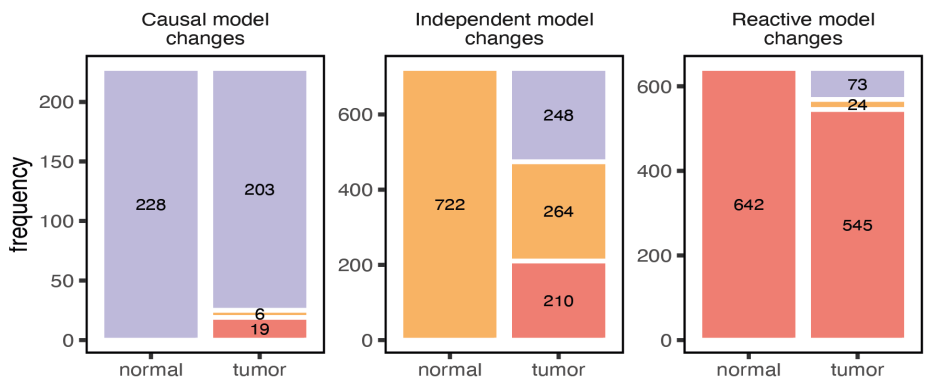

D

Model substitutions for the union between normal and tumor triplets

$(\mathrm{N}=21647)$

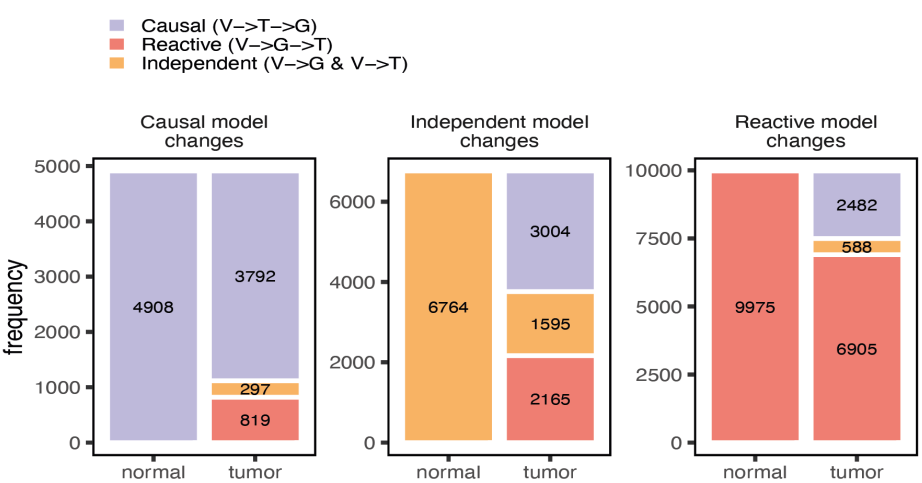

Supplementary figure 20 | Model shifts between normal and tumor. Model shifts for shared triplets between normal and tumor $(A-B)$ where $(A)$ represent the percentage of shared triplets tested that show either a higher posterior probability for the causal, reactive or independent model in tumor and normal. (B) Represents the model shifts from normal to tumor. (C-D) represent the same as A and B but for the union of normal and tumor triplets. In both shared and union, we observe an increase of the causal model in tumor. Independent models in normal and to a smaller extent reactive models are shifting for a causal model in tumor. 


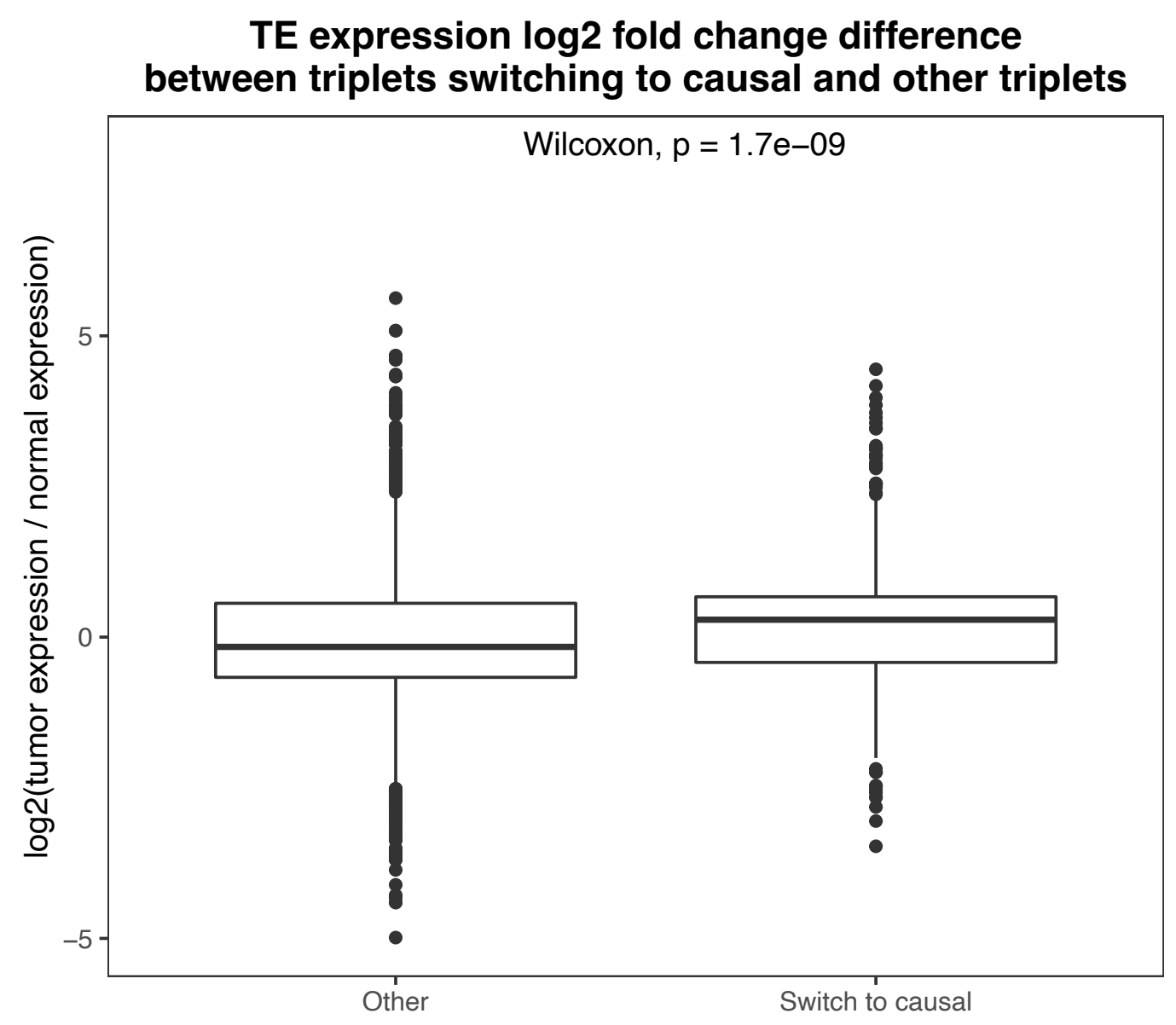

Supplementary figure 21 | log2 fold change of TEs switching to causal versus TEs that do not switch or switch but not to causal. We observe that TEs switching to causal in tumor are significantly more upregulated compared to TEs not switching or switching but not to causal, indicating that this upregulation could explain some of the cases where TEs switch to causal in tumor. 


\section{Supplementary Files}

This is a list of supplementary files associated with this preprint. Click to download.

- SupplementaryTables.xlsx 